\title{
PLASMA RESEARCH
}

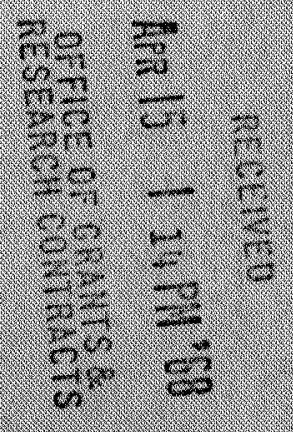

\section{CASE INSTITUTE OF TECHNOLOGY}

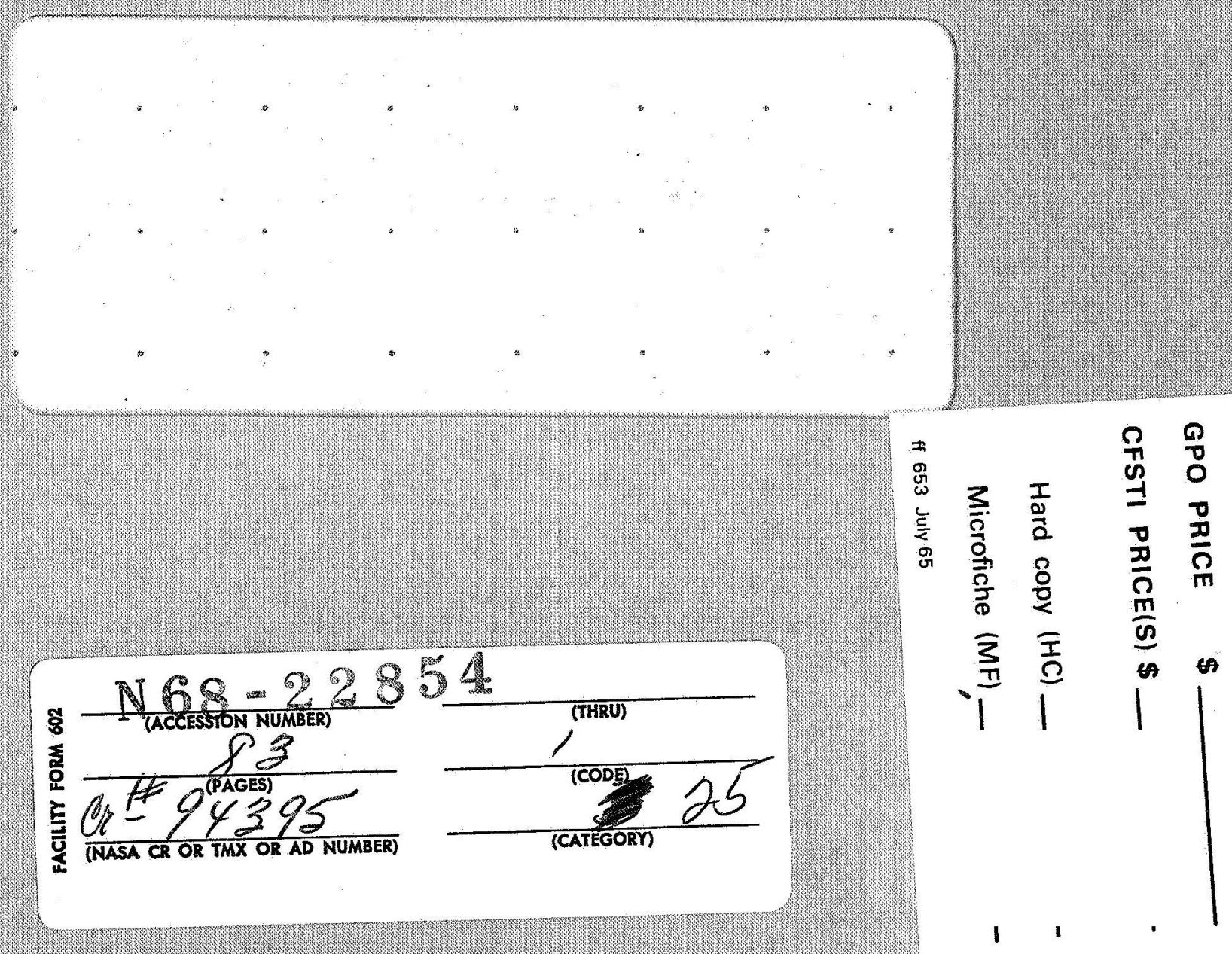

UNIVERSITY CIRCLE • CLEVELAND $6,0 H I O$ 


$$
N 5 G-198
$$

AN EXPERIMENTAL INVESTIGATION OF SHOCK WAVES

IN HIGHLY IONIZED HELTUM ${ }^{*}$

\author{
by \\ George L. Spencer ${ }^{* *}$ \\ Technical Report No. A-43 \\ June 1966
}

*This work supported in part by the National Aeronautics
and Space Administration.
**
North American Aviation Inc. Science-Engineering Fellow. 


\section{ABSTRACT}

An experimental investigation of shock waves moving through a highly fonized gas is reported. The shock waves are generated in an electromagnetic T-tube. The amblent gas, hellum, is highly prelonized by means of an axial current discharge with the shock waves being inftiated during the after-giow period of the preIonization. Measurements have shown that the plasma is in local thermal equilibrium and nearly completely lontzed during the early after-glow perlod of the preionization. Typical temperatures and electron densities are in the order of $22,000^{\circ} \mathrm{K}$ and $3 \times 10^{16}$ electrons $/ \mathrm{cm}^{3}$. Gasdynamic shock waves were studied and the results compared to the theory presented by Jafrrin and Probstein. ${ }^{6}$ It was possible to verify the existence of, and quantitatively measure the thickness of the flirst layer, as predicted by their theory. Magnetohydrodynamic effects were studied via provision of an axial magnetic field. Magnetic field fumps and currents were observed in the shock front and found to be completely stable as long as the shock waves could be observed. It was found that these effects did not appear if either the applied magnetic field or preionization were absent. 


\section{ACKNOWLEDGMENIS}

I would like to thank Professor W. B. Johnson, who served as my mentor throughout the completion of the project. Special appreciation is extended to Dr. Richard Block for many hours of helpful discussions. In addition I am grateful to Mr. Byron Watson for his technical assistance, Mrs. Martha S. Dybas for the typing, and to my wife for reading the manuscript. 
TABLE OF CONTENTS

Page

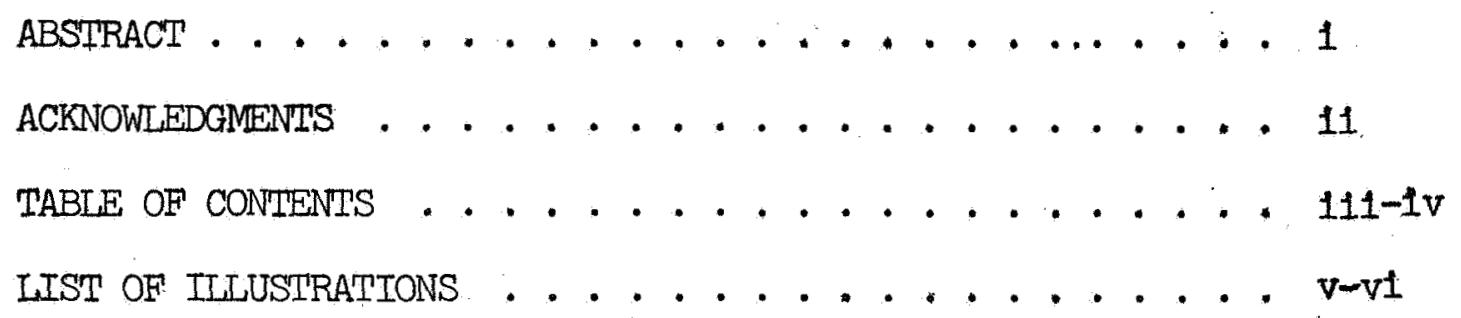

CHAPTER I INTRODUCTION . . . . . . . . . . . 1

CHAPTER II EXPERIMENTAL TECHNIQUES . . . . . . . . .

2.1 Introduction ............ 3

2.2 Magnetic Field Measurements ..... . 9

2.3 Spectroscopic Techniques ....... 9

CHAPTER III PLASMA DIAGNOSTICS . . . . . . . . . . II

3.1 Determination of Temperature . . . . . . 11

3.2 Electron Density Measurements . . . . 13

3.3 Determination of Fractional Ionization and Total Particle Densities..... . 17

3.4 Equilibration Times ........ . . 19

3.5 Plasma Conductivity ........... 21

3.6 Magnetic Diffusion Time......... 21

CHAPTER IV THE PREIONIZATION . . . ......... 23

4.1 Introduction ............ 23

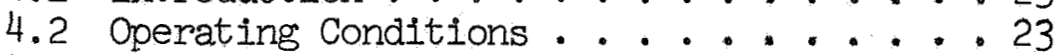

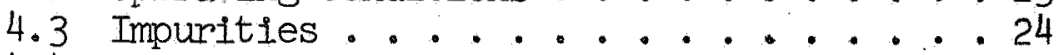

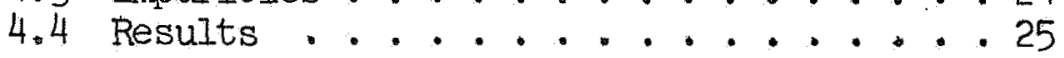

CHAPTER V MAGNETIC FIELD EFHECTS . . . . . . . . 32

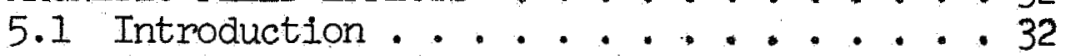

5.2 MHD Parameter Survey ......... . 32

5.3 Current Configuration in the Shock . . . 37

5.4 Summary .......... 43

CHAPTER VI GASDYNAMTC SHOCK WAVES .......... 44

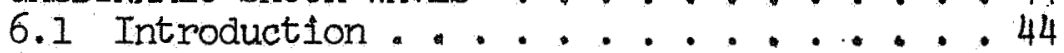

6.2 Shock Thickness ............. 44

6.3 The Sound Speed in PLasma . . . . . . . 55

6.4 Shock Structure for an Ionfing

Shock Wave ............ 59

6.5 Temperatures Bebind the Shock Nave *.. 63

6.6 summary. ................. 64 
CHAPTER VII SUMMARY AND COMMENTS .......... $\frac{\text { Page }}{67}$

APPENDIX A . . . . . . . . . . . $70^{\circ}$

APPENDIX B ................. $73^{\prime}$

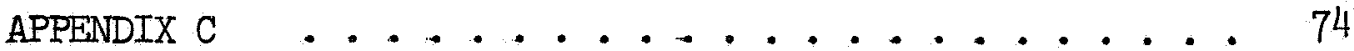

REFERENCES ...................... 75 
LIST OF ILLUSTRATIONS

Figures

Page

2.1 Schematic of the Experimental Apparatus........ 4

2.2 Orientation of the Optical System.......... 5

2.3 Photograph of the Experimental Apparatus . . . . 8

3.1 Ratio of the Total Line and Continuum Intensities as a Function of Temperature for Neutral-Helium . . . 12

3.2 Intensity Ratio of the Lines HeII 4686 Ang, and HeI 5876 Ang, as a Function of Temperature . . . . . 14

3.3 Electron Density as a Function of Temperature and Inital Partial Density $\mathrm{N}_{\mathrm{O}}$. . . . . . . . . 18

4.1 Atom and Continum Radiation During the Preionization Discharge . . . . . . . . . . 26

4.2 Temperature-History of the Decaying Plasma . . . . 27

4.3 Time-History of the Electron Density in the Decaying Plasma . . . . . . . . . . . . 27

4.4 Time-History of the Fractional Ionization in Decaying Plasma............ . . 28

4.5 $N_{0}$ Particle Density History in the Decaying Plasma . . . . . . . . . . . 28

4.6 Radial Variation of Temperature During the Preionization . . . . . . . . . . . 29

5.I Schematic Depicting the Spatial Coordinates . . . 33

5.2 The Variation of the Jump Fields with

Shock Velocity .. . . . . . . . . . 33

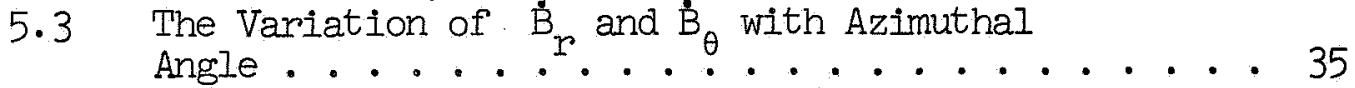

- 5.4 Variation of $B_{\theta}$ as a Function of the Applied

5.5 The Variation of Temperature Behind the Shock as a Function of the Applied Magnetic Field . . . . . 36

5.6 Azimuthal and Radial Jump Fields ......... 38

5.7 A Single Loop Doughnut Current Configuration . . . 40

5.8 Azimuthal Current Patterns . . . . . . . . . 40

5.9 Schematic of Possible Shock Currents . ...... . 41

5.10 The Azimuthal and Radial Components of the Magnetic Field Jumps of a Strong Shock . . . . . . 42

6.1 The Distribution of Flow Variables Though a Medium Strength Shock in a Plasma . . . . . . 46

6.2 Approximate Width of the Electron Thermal Layer as a Function of Electron Density and Temperature . . 48

6.3 Atom and Continuum Intensities for a Medium Strength Shock .. . . . . . . . . . 50 
6.4 Shock Thickness of the First Layer as a Function of Electron Density Behind the Shock. . . . . . 52

6.5 Shock Thickness of the First Layer as a Function of the Electron Density Ahead of the Shock . . . . . 53

6.6 Shock Velocity as a Function of the Energy of the Capacitor Bank . . . . . . . . . . . . . 56

6.7 The Ratio of the Specific Heats, $\gamma$, as a

Function of Temperature .......... 58

6.8 The Square of the Sound Speed as a Function of Temperature................... 61

6.9 Atom and Continuum Intensity Showing the Irregularity in the Shock Front . . . . . . . . .62

6.10 Atom and Continuum Intensity Showing Two Shock Fronts . . . . . . . . . . . . . . 62

6.11 Temperature Behind the Shock as a Function of Shock Velocity .............. 65

A-1 Schematic for Calculation of Radiation From a Plasma .. . . . . . . . . . 71

\section{Tables}

2.I Detailed Information Conceming the Experimental Apparatus . . . . . . . . . 7 


\section{CHAPTER I}

\section{INTRODUCTION}

Gasdynamic and magnetohydrodynamic (MHD) shock waves have been extensively investigated theoretically (-9 $^{1-9}$ These theories utilize certain basic ideas for their solutions; and since the ideas are applied to a new and different physical situation, these applications are always under question. In particular, to obtain the equation of state,the plasma is treated as an ideal gas; and in the equations of momentum and energy transfer between species, the plasma is treated as a gas in which binary collisions take place with the coulomb effects cut off at a radius equivalent to the Debye length. The use of these simplified concepts must always be justified by a comparison to reality of the results predicted by these theories. Even using these simplified ideas the shock theories are not complete because in the practical case the problem is further complicated by such things as radiative transfer and ionization processes, so that it is in general difficult to make experimental verifications.

Both gasdynamic and MHD experiments have been performed ${ }^{10-15}$ where the gas was not initially ionized. The gasdynamic shocks are complicated by the complexity of multiple ionization processes thru the shock, and in the ionizing MHD experiments the agreement between theory and experiment is poor. In either case, if the gas is initially cold, effects of the shock structure present in the 
initially fully ionized plasma are a priori eliminated. In addition, the details of the theories developed for an ionizing shock will not apply to the large body of literature in which shocks propagate in a plasma (e.g., the interstellar medium). Thus it seems desirable to study plasma phenomena in a gas which initially was rendered highly ionized. This may eliminate problems with ionization and permit a more direct comparison between this large body of literature and experiment. 
CHAPTER II.

\section{EXPERIMENTAL TECHNIQUES}

\subsection{Introduction}

The method chosen for the preionization was to run a high current discharge in the gas, using a condenser to supply the needed energy. This method has three distinct advantages: (1) nearly all the energy stored in the condenser is transferred to the gas (as opposed to for example microwave and R.F. methods where the coupling is usually quite poor), (2) the power is quite sufficient to completely ionize the gas and (3) the method is simple. The current in the preionizing discharge was kept as low as possible so that any pinch tendencies would be minimal. Further, the shock waves were observed after the preionizing current had completely stopped, when the plasma, from all available evidence, was quite quiescent. It was decided to use helium because the methods for making temperature and electron density measurements are convenient and well established for this gas. The shock is generated in an electromagnetic shock tube known as a "T" tube, a choice again predicated by the ease of use and the satisfactory results obtained. The characteristics of the "T" tube have been discussed by McLean. 15

A schematic of the experimental apparatus is shown in Figs. 2:1 and 2.2. The operating procedure is as follows: 


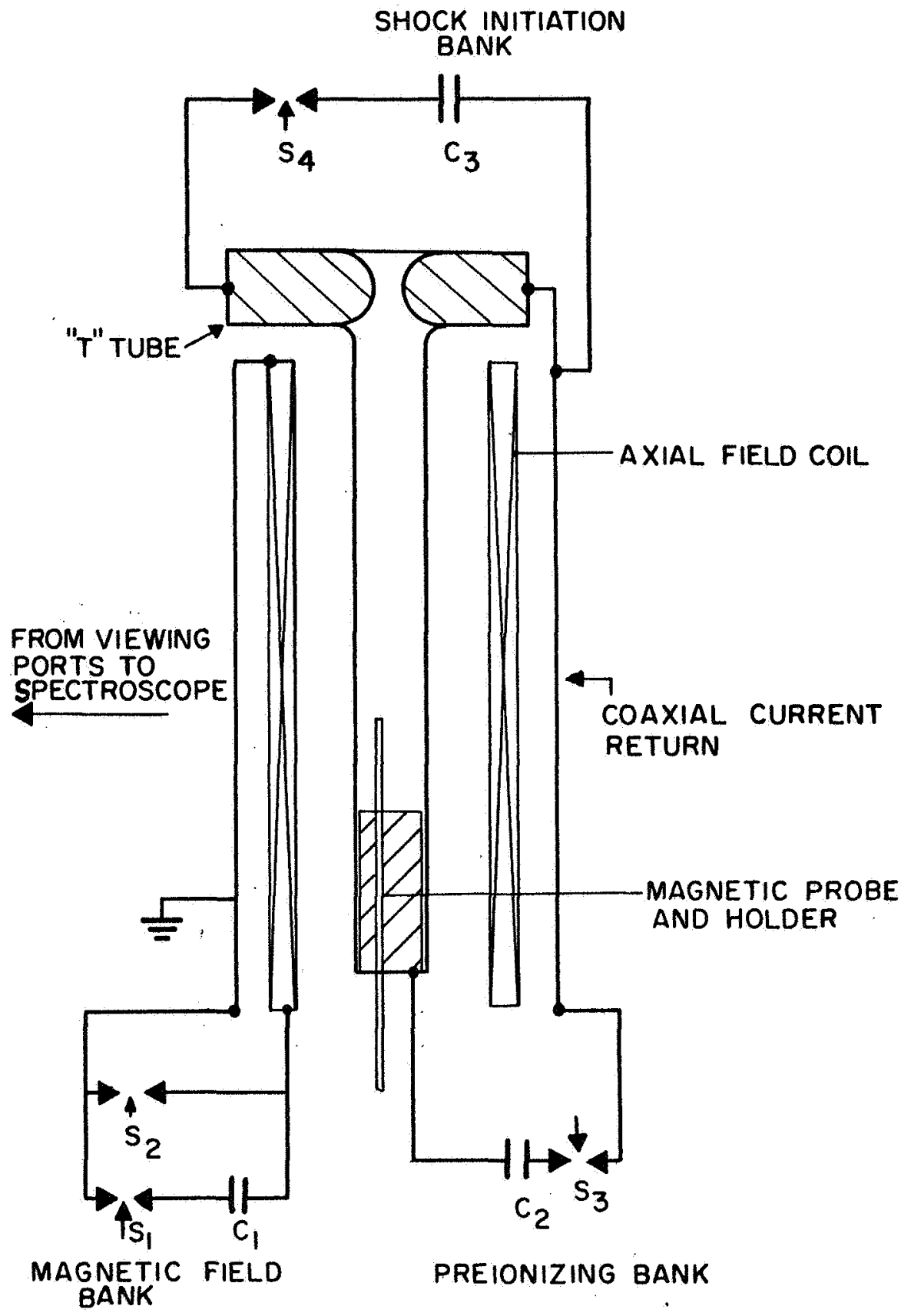

Fig. 2.1 Schematic of the Experimental Apparatus 


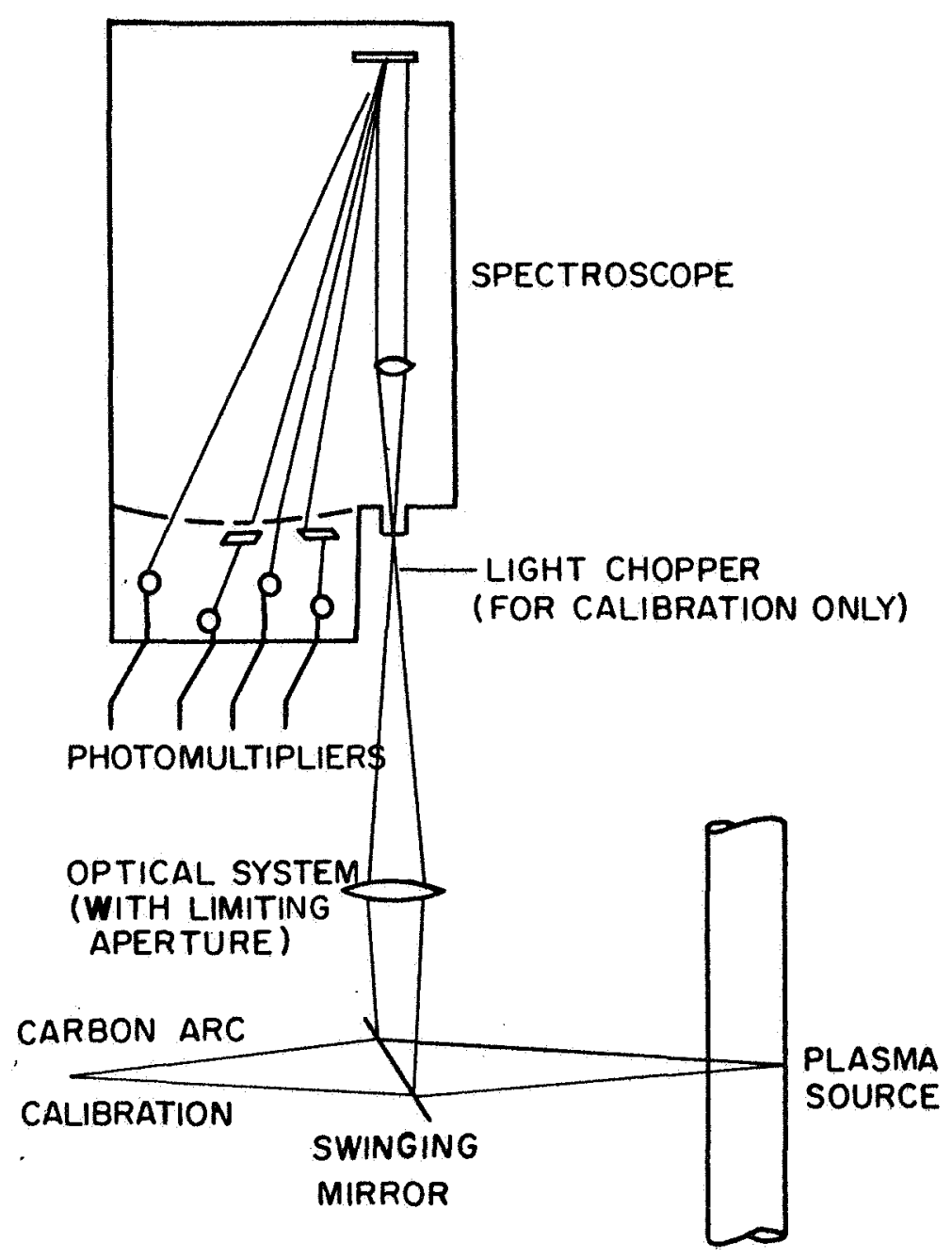

Fig: 2.2 Orientation of the Optical System 
(1) The axial magnetic field is initiated by the discharge of condenser, $\mathrm{C}_{1}$, through the spark gap switch, S-1."

(2) The current in the axial field coil is allowed to increase until it reaches its maximum, and then it is crowbarred by the spark gap switch, S-2, so that the field in the axial coil decays slowly. Magnetic fields up to 1.3 webers $/ \mathrm{m}^{2}$ are available.

(3) The ignitron, S-3, triggers the preionizing bank, $\mathrm{C}_{2}$, which deposits nearly all its energy in the gas, yielding a highly preionized helium plasma.

(4) The ignitron, $s_{4}$, triggers the shock initiation bank, $C_{3}$. The shock wave is generated in an electromagnetic shock tube known as a "T" tube. ${ }^{15}$ The ignitron, $s_{4}$, initiates the discharge such that the disturbance arrives at the viewing port after the current in the prelonizing bank, $\mathrm{C}_{2}$, has completely stopped.

Table 2.1 gives detailed information conceming the components used in Figs. 2.1 and 2.2 . A photograph of the experimental apparatus is shown in Fig. 2.3 .

* Omitted in the case of a gasdynamic shock. 
Table 2.I Detailed Information Concerning the Experimental Apparatus (See Figs. 2.1 and 2.2)

$\underline{\text { Item }}$

1. Shock Tube

2. "T" Electrodes

3. Axial Field Coil

4. $s_{1}, s_{2}$

5. $\mathrm{S}_{3}, \mathrm{~S}_{4}$

6. Magnetic Probe Holder

7. Magnetic Probe

8. $\mathrm{C}_{1}$

9. $\mathrm{C}_{2}$

10. $\mathrm{C}_{3}$

11. Spectrograph

12. Oscilloscope

13. Magnetic Probe Voltage Integrator

14. Spectral Source Osram lamp. glass tube. dispersion.

amplifier

\section{Description}

1" bore, 36" long, Pyrex.

Stainless, hemispherical ends, I" diameter.

\#8 Cu. wire, 150 turns, 36" long, 2.5" Dia., $110 \mu \mathrm{h}, .15 \Omega$.

Vacuum spark gap switches.

Ignitrons. (GE No. GL-7703)

Stainless steel.

3/16" long, 15 turns, enclosed in a 1/8" O.D.

$60 \mu f$, 10KV, 510us ringing period, 3000 joules.

$2 \mu f, 20 K V, 12 \mu$ s ringing period, 450 joules.

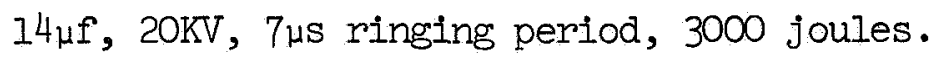

1.5 meter Bausch \& Lomb diffraction grating instrument, model \#12, f/27, $10.4 \mathrm{Ang} / \mathrm{mm}$

Tektronix \#551

Tektronix type "O" plug-in operational. 


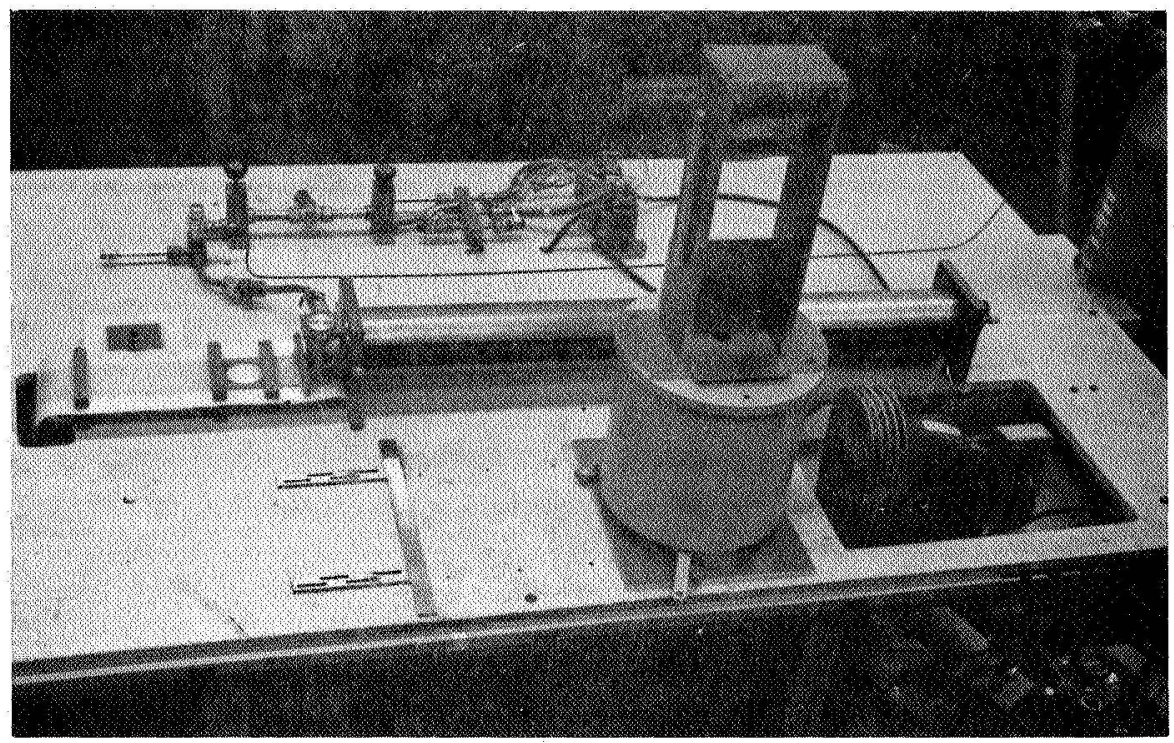

Fig. 2.3a

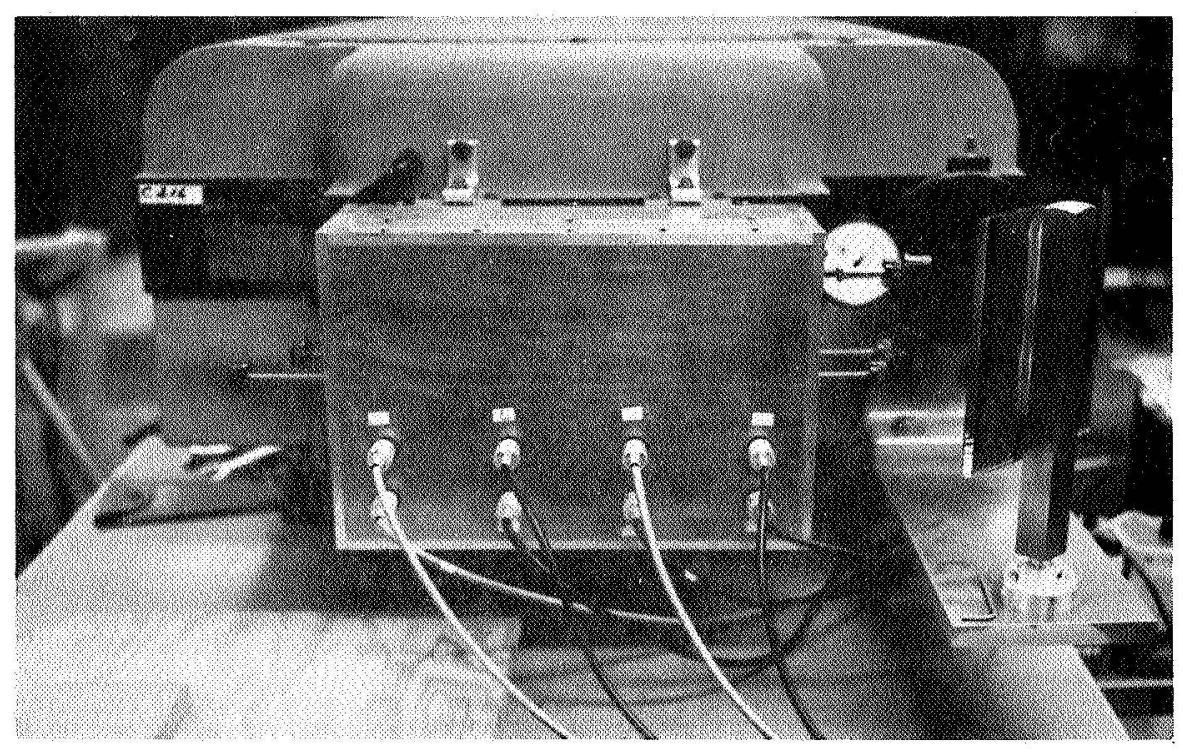

Fig. 2.3b

Fig. 2.3 A Photograph of the Experimental Apparatus.

Fig. 2.3a shows the coaxial current return path (center) with slit for observing the plasma. The " $T$ " is on the left and the magnetic probe holder on the right. The carbon arc lamp is at the lower right. Fig. 2.3b shows the four channel photomultiplier unit mounted on the spectrograph. 


\subsection{Magnetic Field Measurements}

The magnetic field jumps were measured with small pick-up coils located within the plasma. Two or three coils were wrapped within the same volume so that the radial, azimuthal, and axial field jumps could be measured simultaneously. In addition, the probe holder is made so that the probe is variable with respect to its radial, azimuthal, and longitudinal position. The final pick-up coil used was enclosed in a glass tube but exposed coils were also used; no significant discrepancy between the two was noticed. The probe and integrating network were calibrated simultaneously in the tank coil of a $1 / 2$ megacycle Hartley-type oscillator. Thus the entire field recording system was calibrated at a frequency which corresponded to the time behavior of signals observed during the experiment.

\subsection{Spectroscopic Techniques}

The state of the plasma is determined by measuring the atom and continuum radiation intensity from the plasma. Fig. 2.2 shows the method of accomplishing this. The light emitted from the plasma is focused on the entrance slit of the spectrograph. At the exit plane there are four slits which permit only the desired atom, ion, and continuum radiation to reach the photomultipliers. The slits are fixed relative to each other (being milled in a beryllium-copper sheet) but were positioned correctly by use of a helium spectral source. Absolute and relative calibration of the entire system is accomplished by rotating the mirror and using the carbon arc standard (the use of the carbon arc lamp as a standard is discussed 
by Griem ${ }^{16}$ ). During calibration a light chopper, with an "on" time approximating that of the longest signal, was employed. This served as a time-linearity check over the regions of interest. It is necessary, for absolute calibration, to be assured that the limiting aperture of the system is not at the carbon are or plasma source. This was accomplished by a calibrated set of stops at the focusing lens. This set of stops also was used to assure linearity of the system for varying light intensities. Finally, it is assumed that the effective volume of radiating plasma is $A l$, where $A$ is the area focused into the plasma and $l$ is the depth of the emitting volume. It is shown in Appendix A that this is true even though the area cannot be in focus over the entire depth of the plasma volume. 
CHAPTER III

PLASMA DIAGNOSTICS

\subsection{Determination of Temperature}

The electron temperature was determined using the following two methods:

(I) The first method, useful for temperatures in the range of $15,000^{\circ} \mathrm{K}$ to $30,000^{\circ} \mathrm{K}$, is that of measuring the ratio of the intensities of the $\mathrm{HeI} \lambda 5876$ line to the continuum radiation at the same frequency. The continuum intensity is conveniently measured on either side of the line and an interpolation used to give the value at the line position. For complete rigor, correction should be made for the contribution to the continuum by the intensity in the line wings and for the contribution to the line from the underlying continuum, as well as the loss of the line intensity in the wings due to the finite slit width. For this experiment the HeI $\lambda 5876$ line is strong compared to the continuum, the continuum slits are located well away from the line ( 100 Ang), and the exit slit for the atom line is wide enough ( 16 Ang.) so that nearly all the line intensity is included. The omission of these corrections has been estimated to cause less than $1 / 2 \%$ error in the final temperature. The ratio of the HeI $\lambda 5876$ line to the continuum intensity of 100 Ang width is

shown in Fig. 3.1 as given by Griem. ${ }^{16}$ The curve is independent of electron density and the assumptions are that the gas is in local 


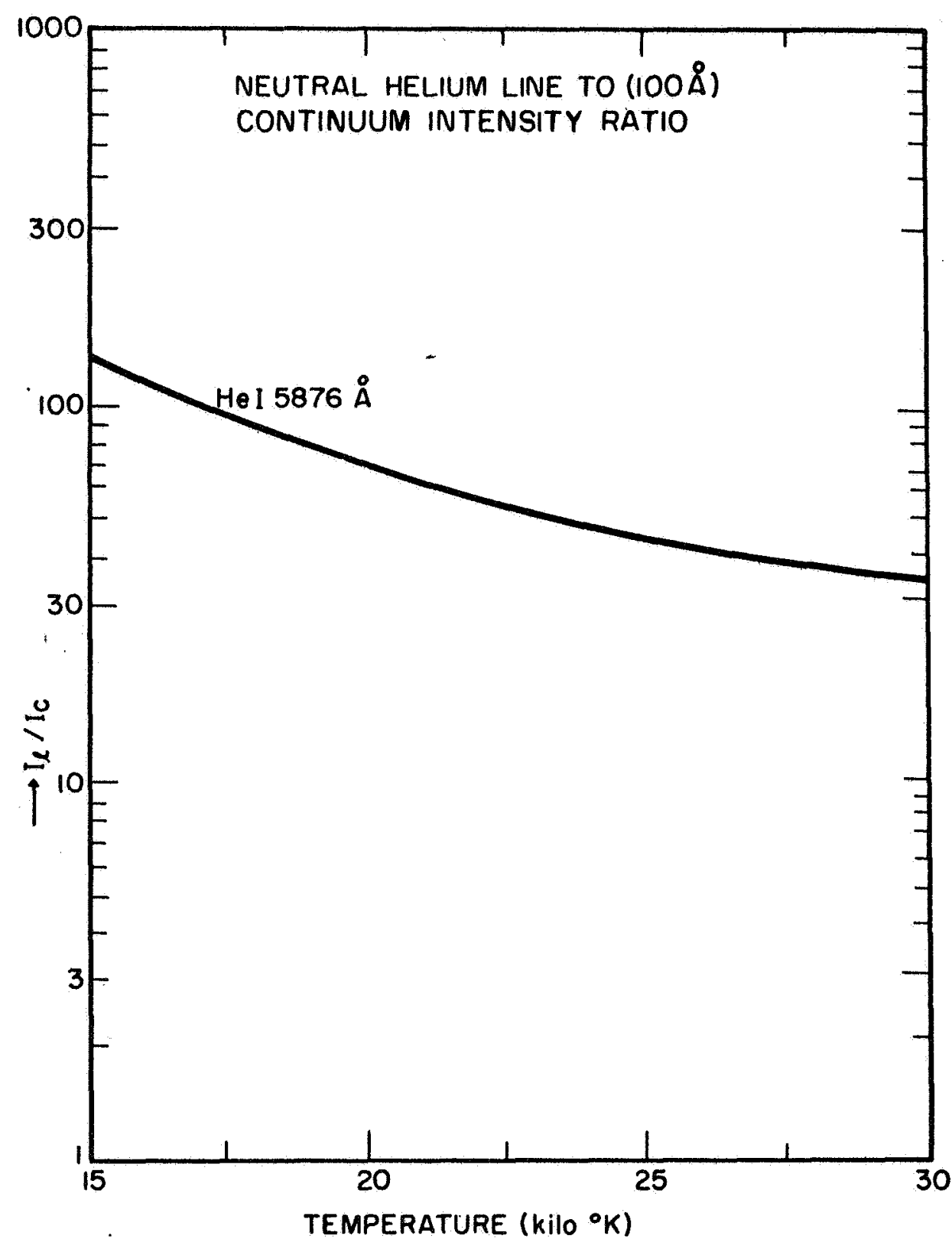

Fig. 3.1 Ratio of the Total Line and Continuum intensities (In 100 Ang. Bands Centered at the Lines) as a Function of Temperature for Neutral-Helium Lines. 
thermal equilibrium (LTE) and optically thin. For the particular case at hand LTE is assured only if the spatial gradients are not too large and the electron density is greater than approximately $10^{15}$ particles per $\mathrm{cm}^{3}$. The fact that the plasma is optically thin for the HeI $\lambda 5876$ Ang.line is indicated in Appendix C.

(2) The second method, useful for temperatures in the range of $35,000^{\circ} \mathrm{K}$ to $75,000^{\circ} \mathrm{K}$, is that of measuring the ratio of the intensities of the HeII $\lambda 4686$ and HeI $\lambda 5876$ lines. The ratio of the intensities of these two lines as a function of temperature is shown in Fig. 3.2 as given by Griem. ${ }^{16}$ In this case the HeI $\lambda 5876$ line is assumed to be in collisional LIE, whereas the HeII $\lambda 4686$ level is assumed to be collisionally populated but radiatively depopulated (corona equilibrium). The condition for the validity of these assumptions is that the electron density per $\mathrm{cm}^{3}$ satisfy the inequality $10^{15} \lesssim \mathrm{N}_{\mathrm{e}} \approx 10^{18}$.

\subsection{Electron Density Measurements}

In principle, if the temperature and total number of particles are known (by setting the ambient pressure), the electron density, during the early quiescent period after the preionizing current has ceased, may be calculated through the use of the Saha equation. However this procedure is never justified a priori since there may exist number density gradients (because of the cooler gas in the pump-out-port, etc.).

The procedure followed for the calculation of electron density 


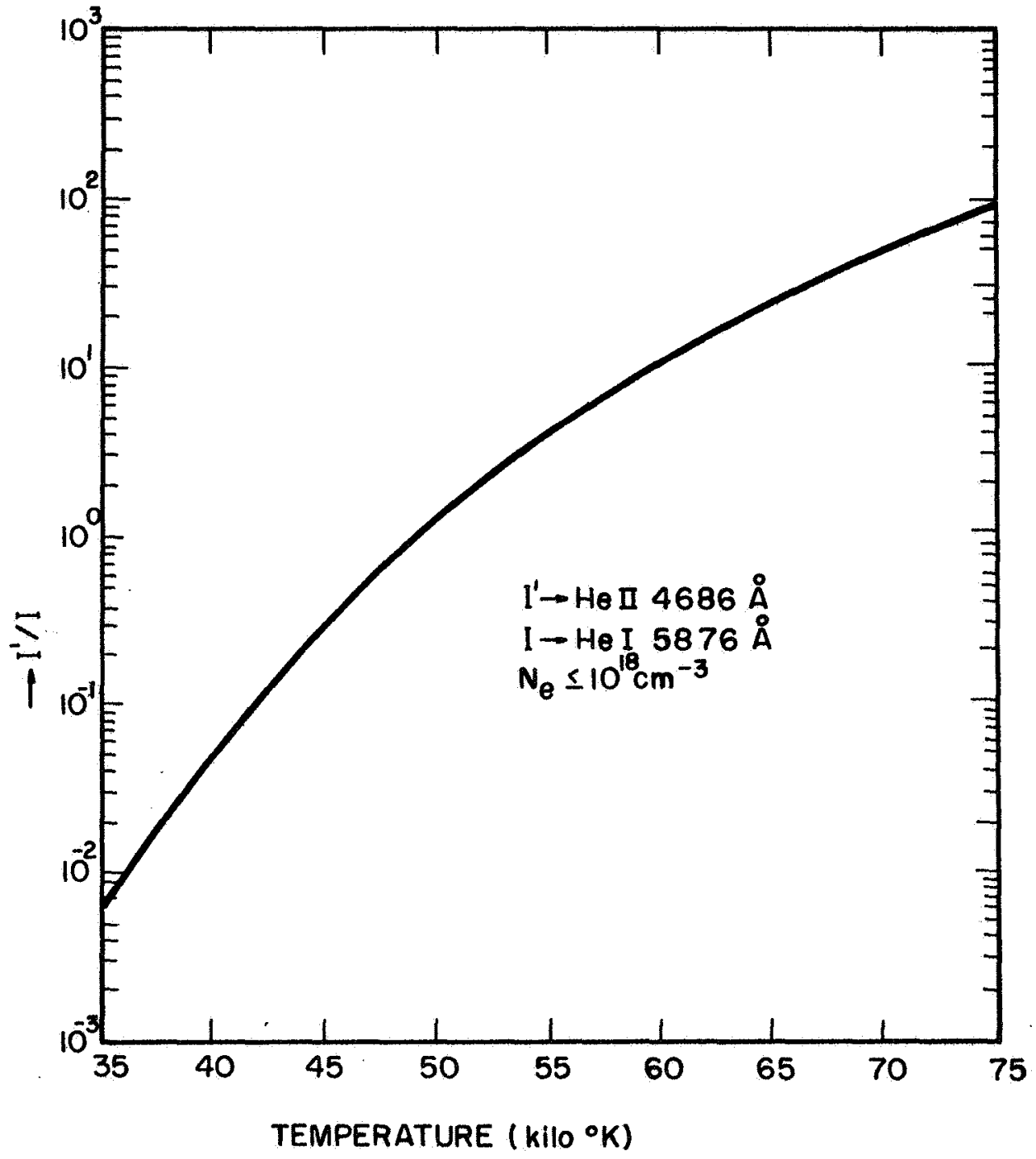

Fig: 3.2 Intensity Ratio of the Lines HeII 4686 Ang.and HeI 5876 Ang as a Function of Temperature. 
is taken from Elton. ${ }^{17}$ There, the continuum intensity, (including the free-free and free-bound contributions) for hydrogen, is given by

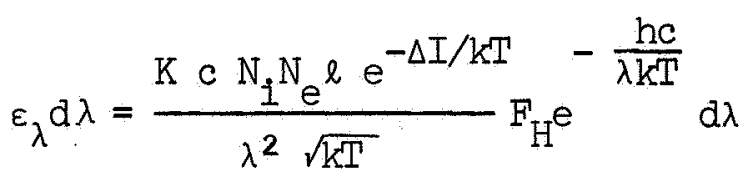

$$
\begin{aligned}
& \text { where } F_{H}=\sum_{n=3}^{n_{g}} \frac{2 E}{k T}<g_{f b}(n)>\frac{e^{E_{n} / k T}}{n^{3}}+\bar{g}_{f f} e^{\frac{E}{\left(n_{g}+1\right)^{2} k T}}-1+g_{f f} \\
& \text { and } \varepsilon_{\lambda}=\text { emissive power in } \frac{e r g i}{\sec -\mathrm{cm}^{3}-\text { steradian }} \\
& K=6.36 \times 10^{-47} \\
& E_{n}=E / n^{2} \\
& \begin{aligned}
<g_{f b}(n)>= & \text { mean free-bound Gaunt factor averaged over the } \\
& \text { sublevels of } n
\end{aligned} \\
& E=\text { ionization energy for hydrogen } \\
& \bar{g}_{\mathrm{ff}}=\text { mean free-free Gaunt factor } \\
& c=\text { velocity of light in vacuum } \\
& \lambda=\text { wavelength at which the continuum is observed } \\
& \mathrm{N}_{e}=\text { electron density } \\
& \mathrm{N}_{i}=\text { ion density } \\
& \Delta I=\text { change in ionization energy due to plasma effects } \\
& \Delta \mathrm{I} \cong .04 \mathrm{ev} \\
& \ell=\text { optical length viewed in plasma } \\
& \mathrm{n}_{\mathrm{g}}=\text { discrete sumation cut off level } \cong \text { or } 7 \text { here, as }
\end{aligned}
$$




$$
\begin{aligned}
& \mathrm{n}=\text { quantum level of atom } \\
& \mathrm{T}=\text { temperature } \\
& \mathrm{k}=\text { Boltzman's constant } \\
& \mathrm{h}=\text { Planck's constant }
\end{aligned}
$$

The units for the above quantities are c.g.s.

Using the above expression, values of Gaunt factors from Griem ${ }^{16}$, and multiplying by 1.1 (see ref. 17) to correct for the fact that the intensity from helium and not hydrogen is desired, gives for $\varepsilon_{\lambda}$ at $2 \mathrm{ev}$

$$
\varepsilon_{\lambda}=1.97 \times 10^{-21} \mathrm{~N}_{e}^{2} l \frac{\text { ergs }}{\sec -\mathrm{cm}^{3}-\text { steradian }}
$$

assuming $\mathrm{N}_{i}=\mathrm{N}_{e}$.

The above expression is an emissive power per unit area and, for calibration purposes, may be directly compared to that of a

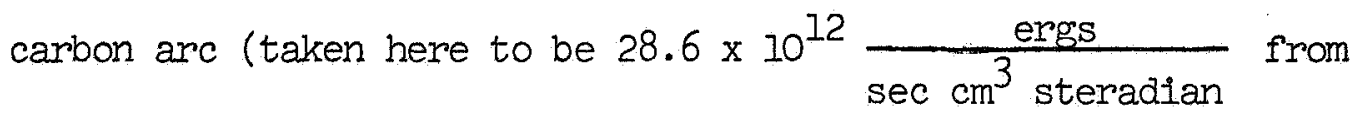
references 18 and 19). Therefore

$$
\mathrm{N}_{\mathrm{e}}=1.66 \times 10^{17}{\sqrt{\mathrm{v}} / \mathrm{N}_{\mathrm{S}}}
$$

where $V_{\mathrm{C}}$ and $V_{\mathrm{s}}$ are the photomultiplier output voltages from the continuum and carbon arc calibration respectively. The above expression for electron density is very insensitive to temperature as noted by Elton ${ }^{17}$ and Cooper ${ }^{20}$, so that over the temperature range of 15,000 to $30,000^{\circ} \mathrm{K}$ the above expression was assumed correct as 
given. This will produce at the extremes an error of approximately $\pm 3 \%$ in $\mathrm{N}_{e}$

3.3 The Determination of Fractional Ionization and Total Particle Density

In addition to the above quantities it is useful at times to determine the fractional ionization, $f$. This is conveniently done with the use of the Saha equation which is for helium

$$
\frac{f^{2}}{1-f}=\frac{4}{N_{0}}\left(\frac{2 \pi m k T}{h^{2}}\right)^{3 / 2} e^{-I / k T}
$$

$$
\text { where } \quad \begin{aligned}
\mathrm{N}_{0} & =\mathrm{N}_{\mathrm{a}}+\mathrm{N}_{\mathrm{e}} \\
\mathrm{f} & =\mathrm{N}_{\mathrm{e}} / \mathrm{N}_{0}=\mathrm{N}_{\mathrm{i}} / \mathrm{N}_{0}=\text { fractional ionization } \\
\mathrm{m} & =\text { electron mass } \\
\mathrm{k} & =\text { Boltzmann's constant } \\
\mathrm{T} & =\text { temperature } \\
\mathrm{h} & =\text { Planck's constant } \\
\mathrm{I} & =\text { ionization energy of } \mathrm{He}(=24.6 \mathrm{ev})
\end{aligned}
$$

The above equation includes an evaluation of the atomic and the ionic partition functions which are taken here to be unity and two respectively. This is nearly true if $\mathrm{T} \leqslant 25,000^{\circ} \mathrm{K}$ which adequately covers the range of interest. Fig. 3.3 shows $\mathrm{N}_{\mathrm{e}}$ (determined from eq. 3.4) as a function of temperature and $N_{\circ}$ (also expressed in terms of the ambient pressure, i.e., $N_{0}=3.5 \times 10^{16}$ atoms $/ \mathrm{cc}$ if $\mathrm{p}=1 \mathrm{~mm}$ at $\left.300^{\circ} \mathrm{K}\right)$. 


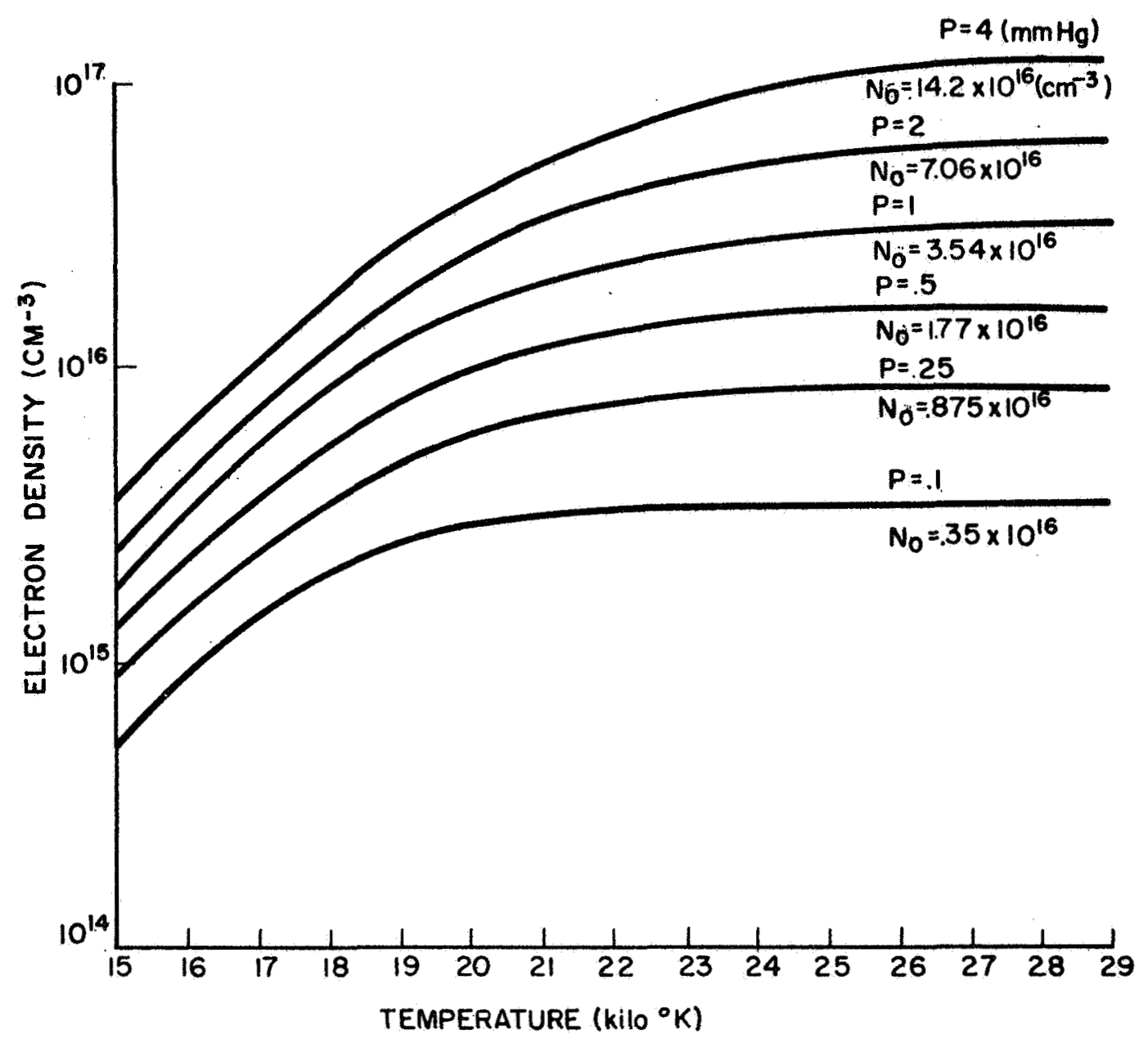

Fig. 3.3 Electron Density as a Function of Temperature and Initial Partial Density No. 
The procedure to be followed would be to determine the temperature from Fig. 3.1 or Fig. 3.2, the election density from eq. 3.3, and then using these values to determine $\mathrm{N}_{0}$ from Fig. 3.3. The fractional ionization is then $\mathrm{N}_{e} / \mathrm{N}_{0}$.

\subsection{Equilibration Times}

The remarks of the preceeding sections only apply if the plasma is in ITE so that one must allow a sufficient time to elapse after gross changes in the plasma occur (i.e., across the shock). The above results depend upon the excitational equilibration time, which according to $\mathrm{Griem}^{16}$ is in the order of .3us for the worst case of equilibrium of the upper levels of HeII . This means that times of this order should be allowed in making use of the HeII $\lambda 4686$ line for measurements. As pointed out by Griem this time is probably too long in the case of electromagnetic shock tubes because the equilibration times are often considerably shortened by radiative excitational and ionization processes. This occurs in the electromagnetic shock tube by radiative transfer from the very hot gas near the tee to the cooler gas in the shock tube. On the other hand, equilibrium is established among the upper levels of HeI and the free electrons in very short times. Since the gas is already highly ionized and all the energy levels are approximately at the same energy (as compared to, say, the energy difference between the $n=1$. and $n=2$ states of HeI and HeII), this time is in the order of $10^{-9} \mathrm{sec}$. Thus the ratio of the HeI $\lambda 5876$ line to the continuum intensity should provide an indication (perhaps not quantitative) of 
the electron temperature even for very short times.

Not directly connected with the previous sections but of interest later is the electron-electron, ion-ion, electron-ion, and electron-neutral ( $t_{e e}, t_{i i}, t_{e i}, t_{e N}$ ) equilibration time, which is the time required to establish kinetic equilibrium between 2 species. . This time as given by Griem $^{16}$ is

$$
\operatorname{t\sim }[\mathrm{N} \sigma \mathrm{V}]^{-1} \mathrm{M} / \mathrm{m}
$$

for two species of number density $N$, cross section $\sigma$, relative velocity $\mathrm{V}$, and mass ratio $\mathrm{M} / \mathrm{m}$ where $\mathrm{M}$ and $\mathrm{m}$ are the heavier and lighter masses respectively. Listed below are these times, using for the cross sections those of Spitzer ${ }^{21}$, assuming a particle density of $10^{16} / \mathrm{cm}^{3}$ and a velocity corresponding to $\mathrm{kT}=2 \mathrm{ev}$.

$$
\begin{aligned}
& t_{e e} \cong t_{e i} \sim 10^{-11} \mathrm{sec} . \\
& t_{i i} \simeq 10^{-9} \mathrm{sec} . \\
& t_{e i} \cong t_{e N_{a}} \sim 10^{-7} \mathrm{sec} .
\end{aligned}
$$

At first it might be thought that $t_{\mathrm{eN}_{\mathrm{a}}}$ should be the longer time. This would be true except that the mean life time of a neutral atom is short $\left(<10^{-7}\right.$ sec. $)$ due to charge exchange, so that the two times are approximately equal. 


\subsection{Plasma Conductivity}

The plasma conductivity is useful for the determination of the magnetic diffusion time. According to Spitzer ${ }^{21}$ the conductivity for a singly ionized gas is

$$
\sigma=\frac{6.2 \times 10^{6} \mathrm{~T}^{3 / 2}}{\ln \Lambda}
$$

$$
\text { where } \begin{aligned}
\sigma= & \text { conductivity in }[\mathrm{ohm}-\mathrm{cm}]^{-1} \\
T= & \text { temperature in } \mathrm{KEV} \\
\ell \mathrm{ln} \Lambda= & \text { a slowly varying function of temperature and } \\
& \text { electron density }
\end{aligned}
$$

Typically for this experiment $\ell n \Lambda=5$ and $k T=2 e v$, so that $\sigma \cong 10^{2}[\mathrm{ohm}-\mathrm{cm}]^{-1}$.

\subsection{Magnetic Diffusion Time}

Since a discharge current is used to generate the plasma, and magnetic probes are used to measure magnetic field jumps, it is natural to inquire as to whether the magnetic fields generated in the preionization discharge can diffuse out of the plasma so as to leave a relatively fleld-free region for the shock, and whether the field can penetrate the probes in times of interest. The diffusion time for magnetic fields (or currents) is

$$
t \cong 10^{-8} \cdot \mathrm{dL}^{2} \quad \mathrm{~L} \text { in } \mathrm{cm}
$$

where $t$ is the time in seconds required for the field or current to drop to $1 / e$ of its initial value. 


$$
\begin{aligned}
\sigma & =10^{2}[\mathrm{ohm}-\mathrm{cm}]^{-1} \\
L & =\text { a dimension of interest in the plasma. }
\end{aligned}
$$

In this experiment the shock tube radius $\cong 1 \mathrm{~cm}$ so that the diffusion time is in the order of $1 \mu s$, and the preionizing current ceases from 0 to 10us before the shock arrival. Therefore, since the measured magnetic field $₹ 10$ gauss when the current stops, the plasma fields should be well below 1 gauss at the time of shock arrivad.

For the magnetic probes $\mathrm{L} \sim 1 / 4 \mathrm{~cm}$ and $t<10^{-7} \mathrm{sec}$. This is in general shorter than times of interest. 
CHAPTER IV

\section{THE PREIONIZATION}

\subsection{Introduction}

The objective of the preionization is the production of a reasonably quiescent highly ionized plasma. Numerous authors have mentioned observing plasma luminescence for long periods in excited gases. In particular, Cooper ${ }^{20}$ has completed a study of decaying hydrogen in which the decaying plasma is observed for times of the order of hundreds of microseconds. In the present study plasma Iuminescence was easily observed 500us after the discharge had ceased, but of primary concern is the determination of the state of the plasma just after the current has stopped, when the gas is highly ionized and in a quiescent state.

\subsection{Operating Conditions}

The preionization is accomplished by allowing a high current to flow through the entire length of the shock tube, as mentioned in Chapter II. This method has the advantage of efficient coupling of the energy of the capacitor into the plasma. By calculation, using the value of $\sigma$ obtained in Section 3.5, and by experimental techniques, $75 \%$ of the energy is easily transferred to the plasma. Typical of operating conditions are ambient pressures of $.5 \mathrm{~mm}$ to $4 \mathrm{~mm}$, bank voltages up to $15 \mathrm{KV}$ ( 200 Joules), and initial currents in the order of 12 to 14 kiloamps. It is not desirable to have the currents too large because of the formation of plasma instabilities. 
It was found that the higher ambient pressures produced a much more radially uniform plasma. Indeed, at lower pressures the temperatures calculated by the two methods of Section 3.1 were in violent disagreement during the time of current flow, $\left(240,000^{\circ} \mathrm{K}\right.$ with the ion line, $25,000^{\circ} \mathrm{K}$ with the atom line) indicating non-equilibrium, whereas at higher pressures (2 $\mathrm{mm}$ to $4 \mathrm{~mm}$ ) this effect was not observed.

\subsection{Impurities}

The use of spectroscopic tools for plasma diagnostics demands a system reasonably free from impurity gases, vapors, and readily vaporized solids. The latter two impurities are the serious offenders (since the admitted helium is pure to better than 1 part in $10^{5}$ ) and it was observed that after long periods of firing ( 1000 shots) a metallic deposit from the electrodes would build up to a distance of about 6" from the tee. In addition, a yellowish deposit would form over the entire confines of the tube. This material, it is assumed, is vacuum grease and/or pump oil ejected during preionization and subsequent firing of the shock tube, For this reason the tube was cleaned frequently.

Spectrographic data was taken (up to $~ 250$ shots per photograph) to get an idea of the impurities present so that the photomultiplier slits (particularly the continuum slits) could be located outside the impurity lines. On the 50 shot photograph the impurity lines were numerous but weak. The impurities could not be identified, 
probably due to the slow speed and resolution of the spectrograph. Finally, to be certain that no impurity lines were buried in the continuum, a wide traverse ( 250 Ang) of the continuum slits, and a comparison of the time-history of the observed light intensity (from shot to shot during the time of preionization) was made. No significant differénces were found. It is very unlikely that different impurity lines would have similar time-histories (i.e., a number of lines would have to be of nearly the same intensity over a wide temperature range), so that with the agreement of the intensity of the continuum positions it was taken that there were no underlying impurity lines.

\subsection{Results}

Fig. 4.1 shows the time-history of the radiation from the plasma at an ambient pressure of $4 \mathrm{~mm}$ and a bank voltage of $14 \mathrm{KV}$. The upper trace is the HeI $\lambda 5875$ line and the lower trace is the nearby continuum. In Fig. 4.1a, the current had completely stopped 40us after initiation since the ignitron stopped conducting at this time. Fig. 4.1b is similar to Fig. 4.1a, but with the time scale being expanded and delayed to show the region in which the measurements were taken. The preionizing current stopped at $t=12 \mu \mathrm{s}$ in Fig. 4.1b.

Figs. 4.2 through 4.6 show the computed time-histories of pertinent plasma parameters. Fig. 4.2 shows temperature versus time. The observed temperatures are in the order of $23,000^{\circ} \mathrm{K}$ to 


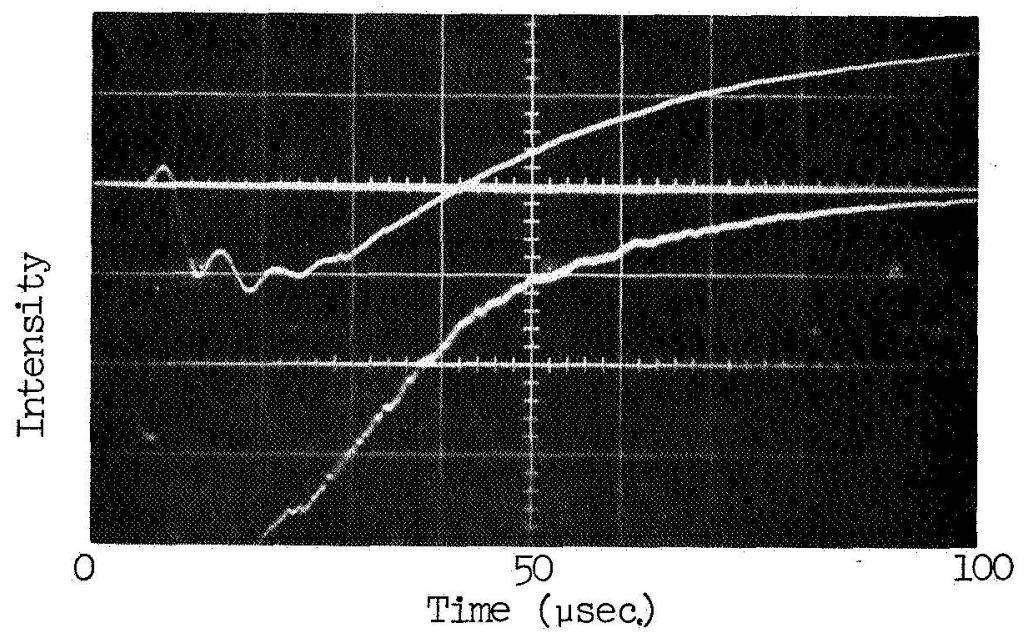

Fig. $4.1 \mathrm{a}$

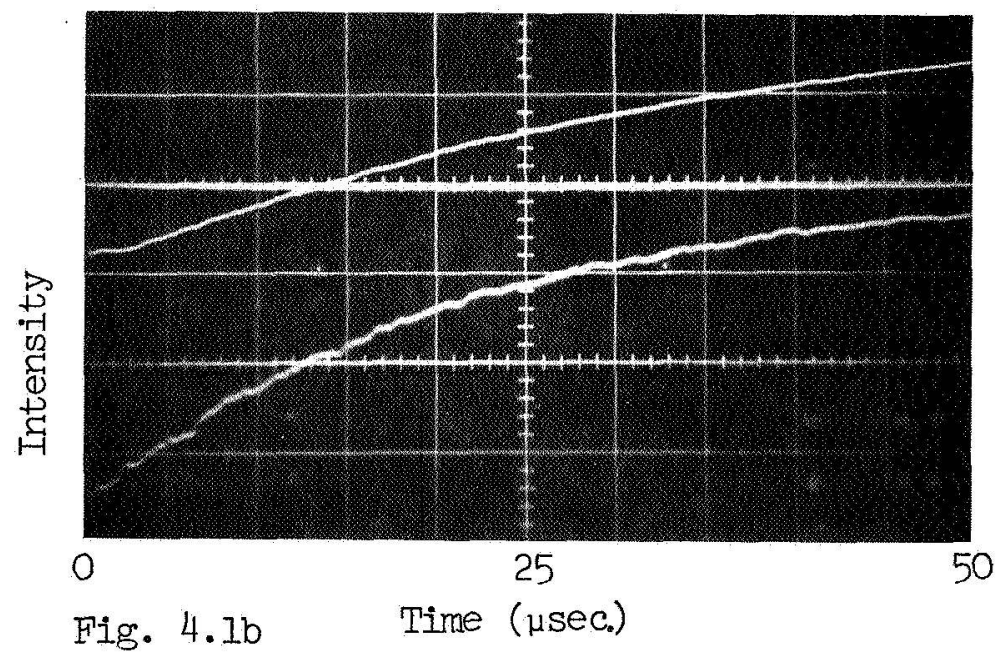

Fig. 4.1 Atom (Upper Trace) and Continuum (Lower Trace) Radiation During the Preionizing Discharge. Fig. 4. Ib is an expansion of Fig. 4. la over the region in which shocks are observed. 


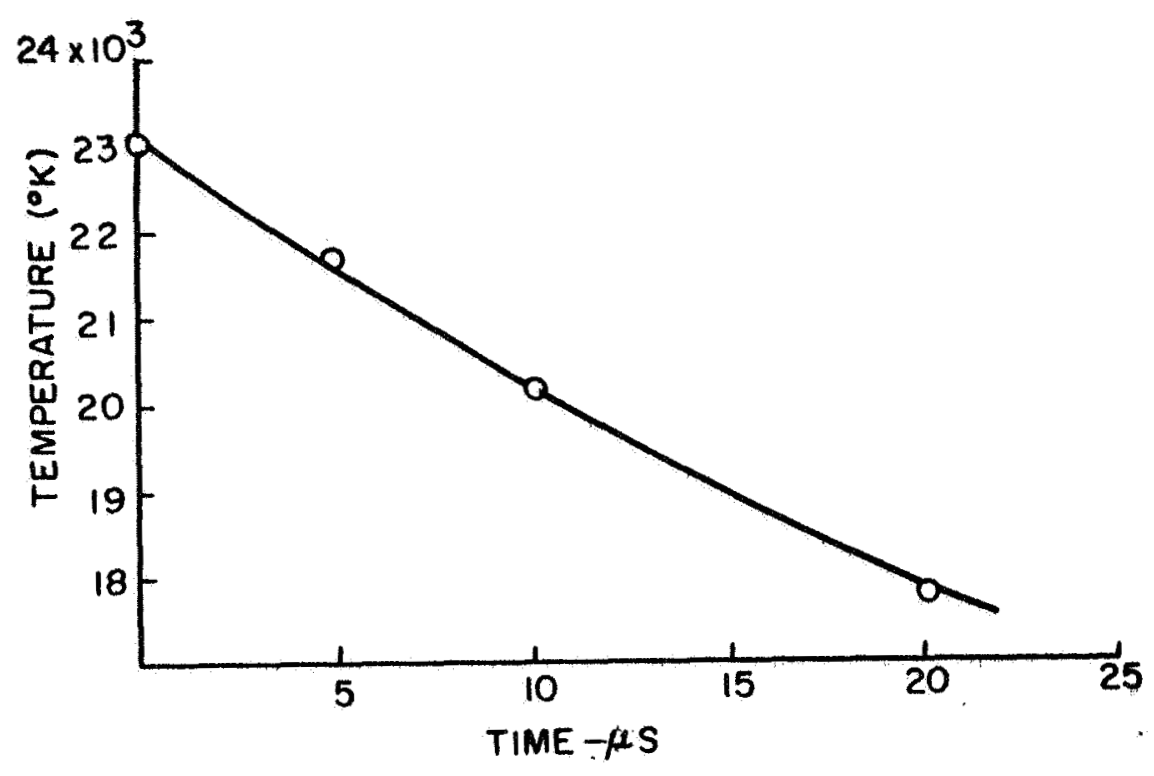

FIg. 4.2 Temperature-History of the Decaying Plasma

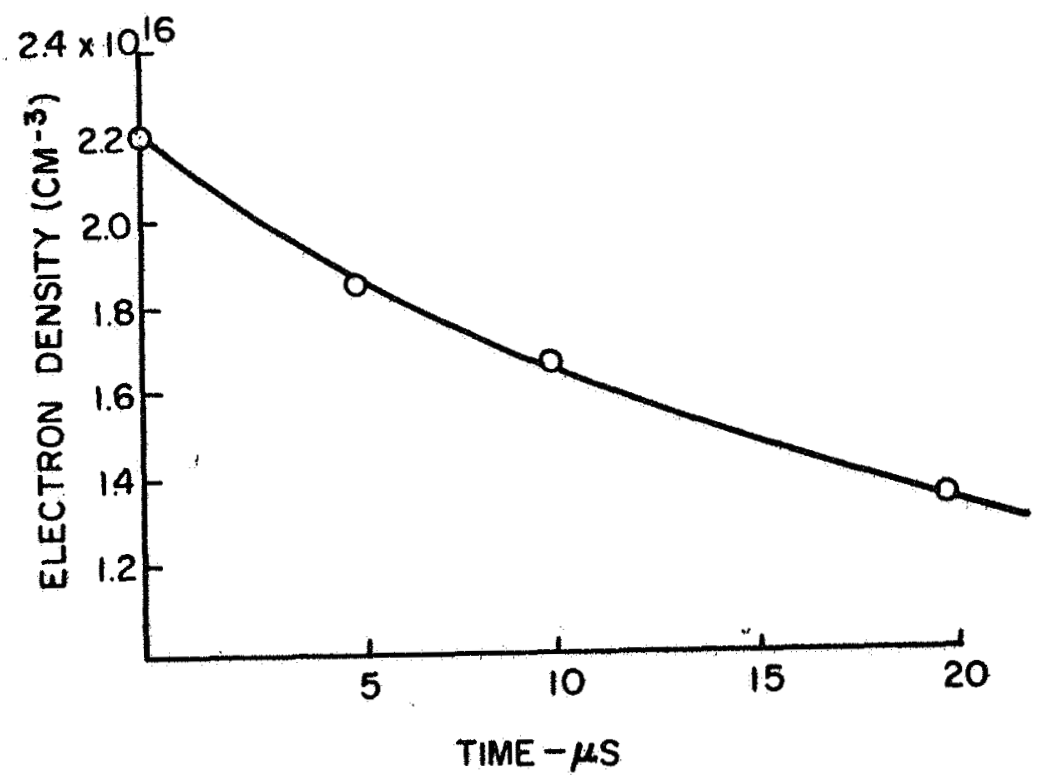

F1g. 4.3 Time-History of the Electron Density in the Decaying Plasma 


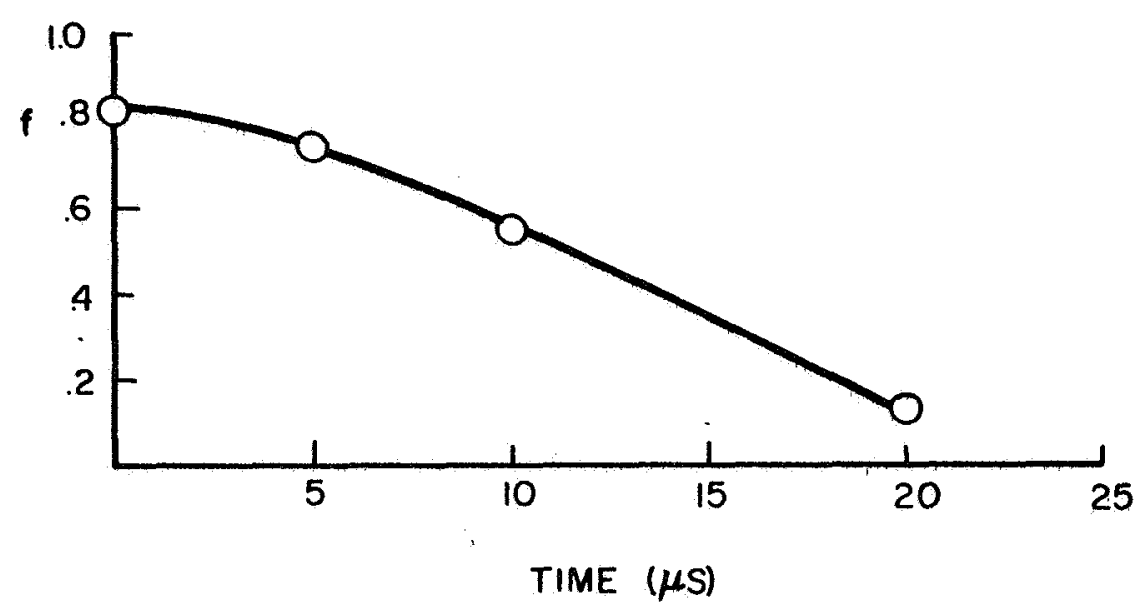

Fig. 4.4 Time-History of the Fractional Ionization in the Decaying Plasma.

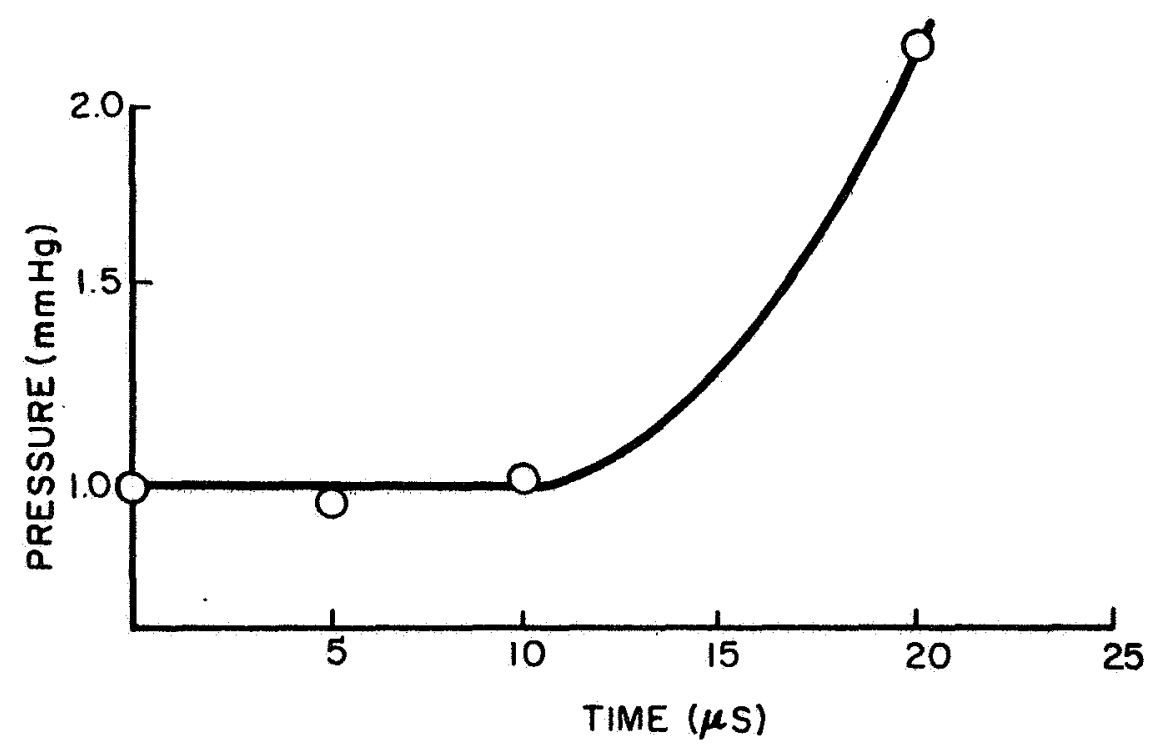

Fig. 4.5 $\mathrm{N}_{\mathrm{O}}$ Particle Density History in the Decaying Plasma, Plotted in Terms of Ambient Pressure. 


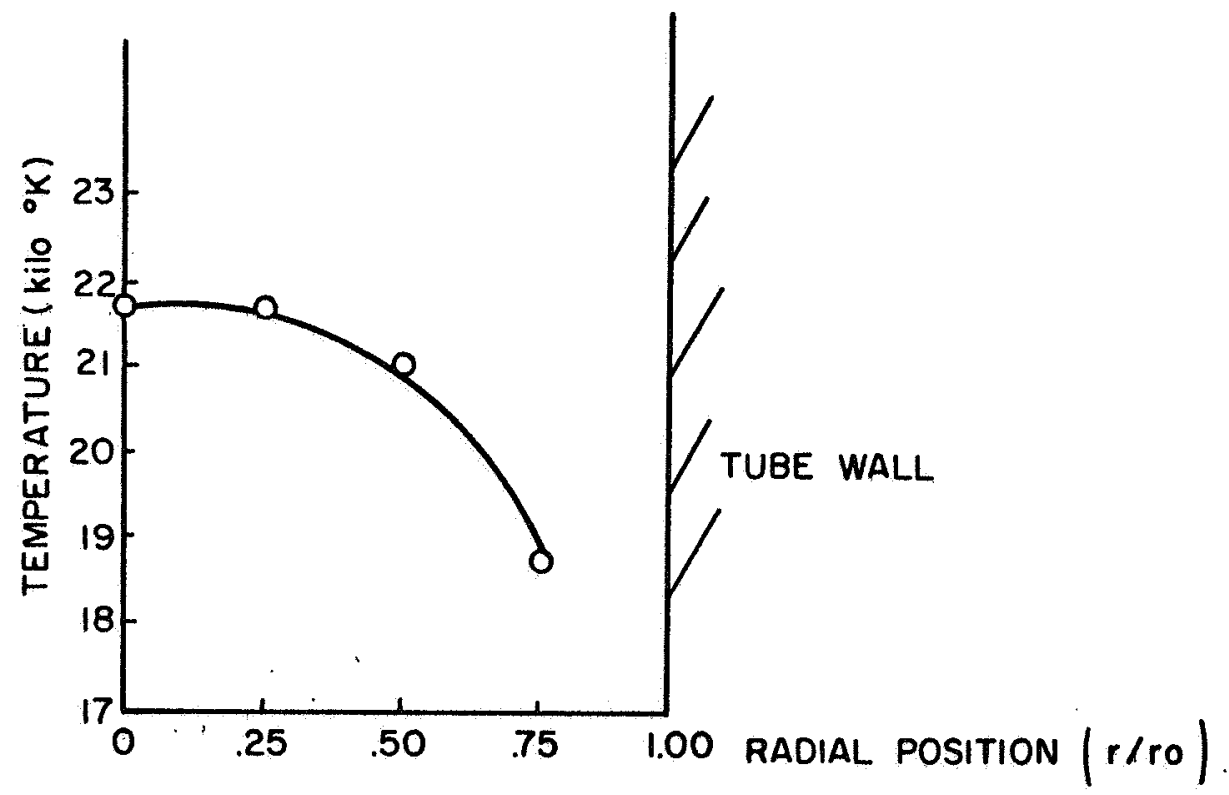
Fig. 4.6 Radial Variation of Temperature During the
Preionization (Taken at $t=5 \mu \mathrm{sec}$ ) 
$18,000^{\circ} \mathrm{K}$, with a decay rate of $250^{\circ} \mathrm{K} / \mu \sec (\sim 1 \% \mu \mathrm{s})$. Fig. 4.3 shows electron density as a time-function. Densities are extremely high (as compared to microwave and glow preionization) being in the order of $10^{16} / \mathrm{cm}^{3}$ with a decay of $2 \% / \mu \mathrm{sec}$. Fig. 4.4 shows that the gas is highly ionized, with useful ionization levels in the order of 85\%. Fig. 4.5 shows the time-history of the particle density, $\mathrm{N}_{0}$, plotted in terms of ambient pressure. The pressure is at first a factor of $5(0.8 \mathrm{~mm})$ lower than ambient and then begins a sharp rise at later times. Due to the volume of gas not being heated (pump-out port, etc.) and the long times of heating $(240 \mu \mathrm{s})$ rough estimates indicate that this lower pressure is not unreasonable. The later rise in pressure is somewhat surprising at first, but could be caused by gas streaming back into the tube, or release of adsorbed gas from the walls. Also likely, however, is the fact that the electron density and temperature are, at later times, too low for LTE, especially near the tube walls. In this case then the temperature computations are no longer valid. Thus, there is no reason to doubt the measurements at earlier times, or electron density measurements at earlier or later times (since this measurement is rather insensitive to either temperature or ITE variations). Finally, Fig. 4.6 shows the radial variation of temperature across the tube. Normally one should measure the intensity in slices across the tube and then perform an Abel transform ${ }^{16}$ to obtain $T(r)$. However, the contribution to the ratio of line to continuum intensities is heavily weighted by the 
hotter regions of the plasma because both the line and continuum strengths increase rapidly with temperature in this temperature region. If a negative temperature gradient exists, most of the contribution from a slice would come from that part which was closest to the tube center. Thus one would not be too much in error by simply taking a slice across the tube at a distance $r$ from the tube center and plotting this as $T(r)$. Fig. 4.6, then, is somewhat pessimistic in that $T(r)$ aetually does not fall off quite so steeply. The fall-off as measured is about $15 \%$ from the center to $3 / 4$ radius.

In surmary, the preionizing discharge produces a highly ionized, reasonably quiescent plasma. The rate of decay of the plasma as compared to equilibration times indicates that the plasma is in ITE at least during the earlier decay times. The plasma is much more uniform and more highly ionized at higher pressures. Perhaps the most disturbing feature is the radial temperature gradient; while in itself not too serious, it implies a gas with low ionization near the walls (recall again, however, that Fig. 3.7 is too pessimistic). This could probably be improved by stopping the current at an earlier more appropriate time, as well as using even higher pressures where diffusion cooling to the walls is less important. As discussed in Section 3.6 frozen-in fields due to the discharge current should not be significant. The results obtained here are in agreement with those of Cooper ${ }^{20}$, although his primary concern was the state of the plasma at later times in the after-glow. 
CHAPTER V

MAGNETIC FIETD EFHECTS

\subsection{Introduction}

The provision of a longitudinal field gives rise to the possibility of studying MHD effects. It was hoped initially that the "T" tube would produce an approximately planar shock wave so that the results could be compared with one-dimensional shock wave theory and, in particular, to determine if the collisionless approach

is valid: (see e.g. Longmire ${ }^{1}$ or Kantrowitz and Petschek ${ }^{2}$. However, the shock formed is by no means planar, but has complete axial symmetry. Thus planar shock theory does not apply and unfortunately the axially symmetric case has not been solved, so that the remarks of this section are necessarily largely qualitative, and to some extent even characteristic of the "T" configuration. Nevertheless it is felt that the results are well worth reporting - they bear on stability problems and future MHD experiments.

\subsection{MHD Parameter Survey}

Figure 5.1 shows the coordinate system used to describe the magnetic fields. The steady axial field, $B_{a}$, is variable up to 13 kilo-Gauss. The field jumps, $B_{A}, B_{r}$, and $B_{z}$ are measured in the azimuthal, $(\hat{\theta})$, radial, $(\hat{r})$, and longitudinal $(\hat{z})$ directions at a point approximately $50 \mathrm{~cm}$ from the "T" . The magnetic field jumps which were observed were small compared to the applied fields, being 


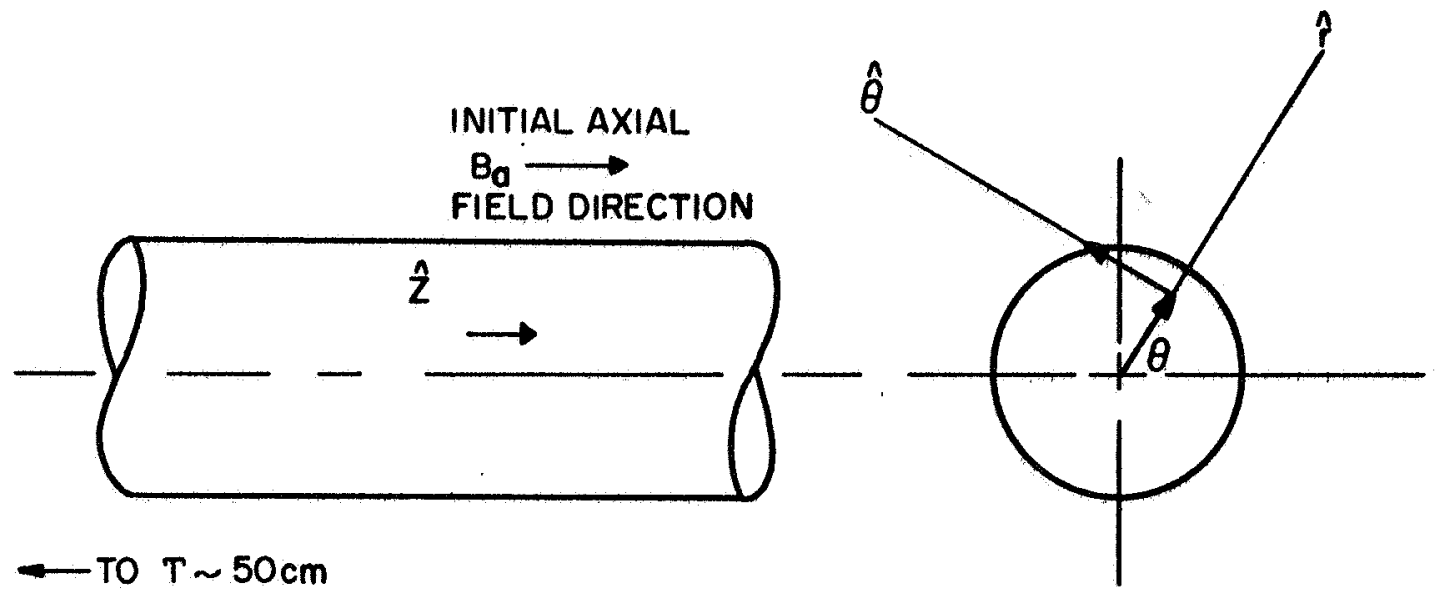

Fig. 5.1 Schematic Depicting the. Spatial Coordinates Referred to in the Text.

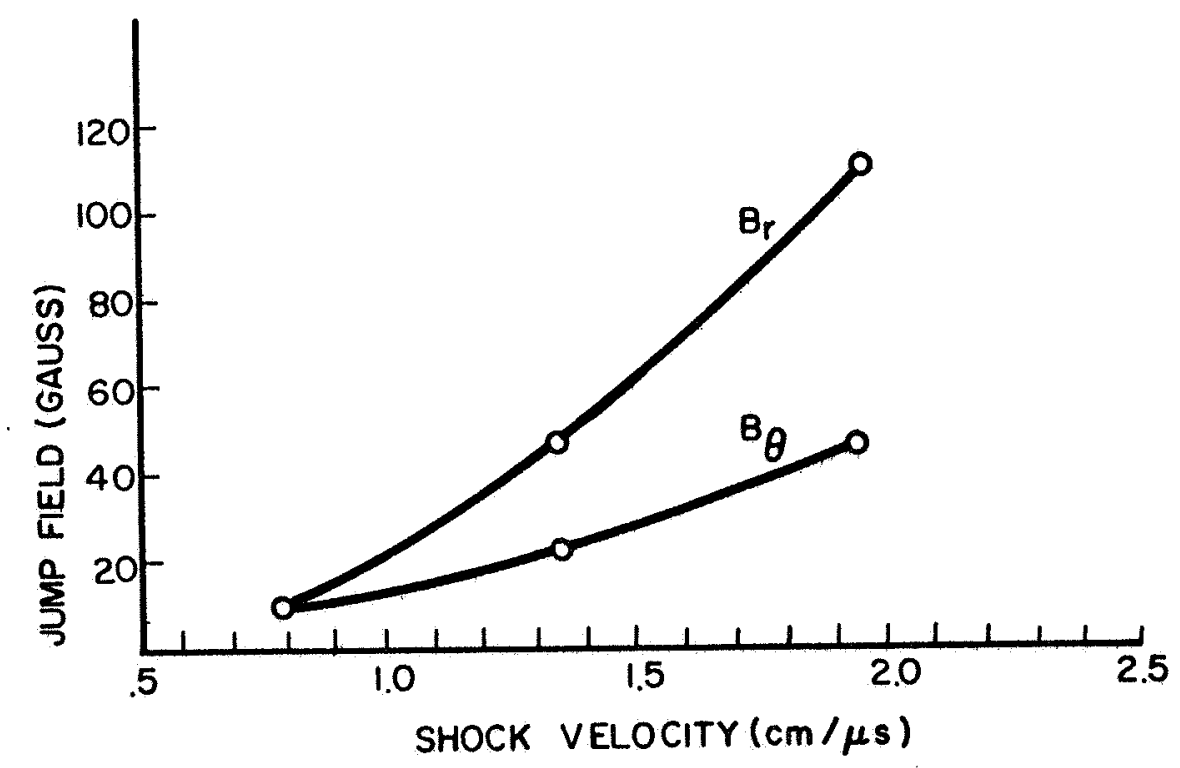

F1g. 5.2 The Variation of the Jump Flelds with Shock Velocity. $\left(B_{a}=4: 8 \mathrm{kilo-Gauss,} r=3 / 16^{\prime \prime}\right)$. 
in the order of 1 to 10 percent. Fig. 5.2 shows the jump field $B_{r}$ and $B_{\theta}$ plotted as a function of shock velocity. Notable is the fact that the radial jump increases faster than the azimuthal jump. Mentioned earlier is the complete azimuthal symmetry of the B fields. Specifically, the value of $B_{\theta}$ or $B_{r}$ measured at a fixed radius is independent of $\theta$. As an illustration of this Fig. 5.3 shows $\dot{\mathrm{B}}_{r}$ (upper trace) and $\dot{\mathrm{B}}_{\theta}$ ( (lower trace) for four values of $\theta\left(\theta=0^{\circ}, 90^{\circ}, 180^{\circ}, 270^{\circ}\right)$. Slight variations occur in the maximum amplitude $(u+10 \%)$ which are probably mostly due to probe positioning error, but in general the reproducibility was excellent. These photo's are indicative of the fact that, in general, individual muns were highly repeatable. Fig. 5.4 shows the variation of $B_{\theta}$ as a function of the applied magnetic field for several shock velocities. Noteworthy is the increase of , and then the decrease of, $B_{\theta}$ with $B_{a}$ for the lower shock velocity. At higher velocities $B_{\theta}$ tapers off but does not decrease, although it appears that if $\mathrm{B}_{\mathrm{a}}$ were increased further the effects would be similar to the low velocity case. (this would agree with planar switch-on shock theory). Fig. 5.5 shows the effect of the applied field on the temperature behind the shock. It is seen that the effect is small with the field causing only a slight increase in temperature.

The above description has ignored the electron density and fractional ionization as a parameter. It was found that, within experimental error, the field jumps are independent of electron density (over a factor of 4 ) if the shock velocity, $V_{S}$, is the 

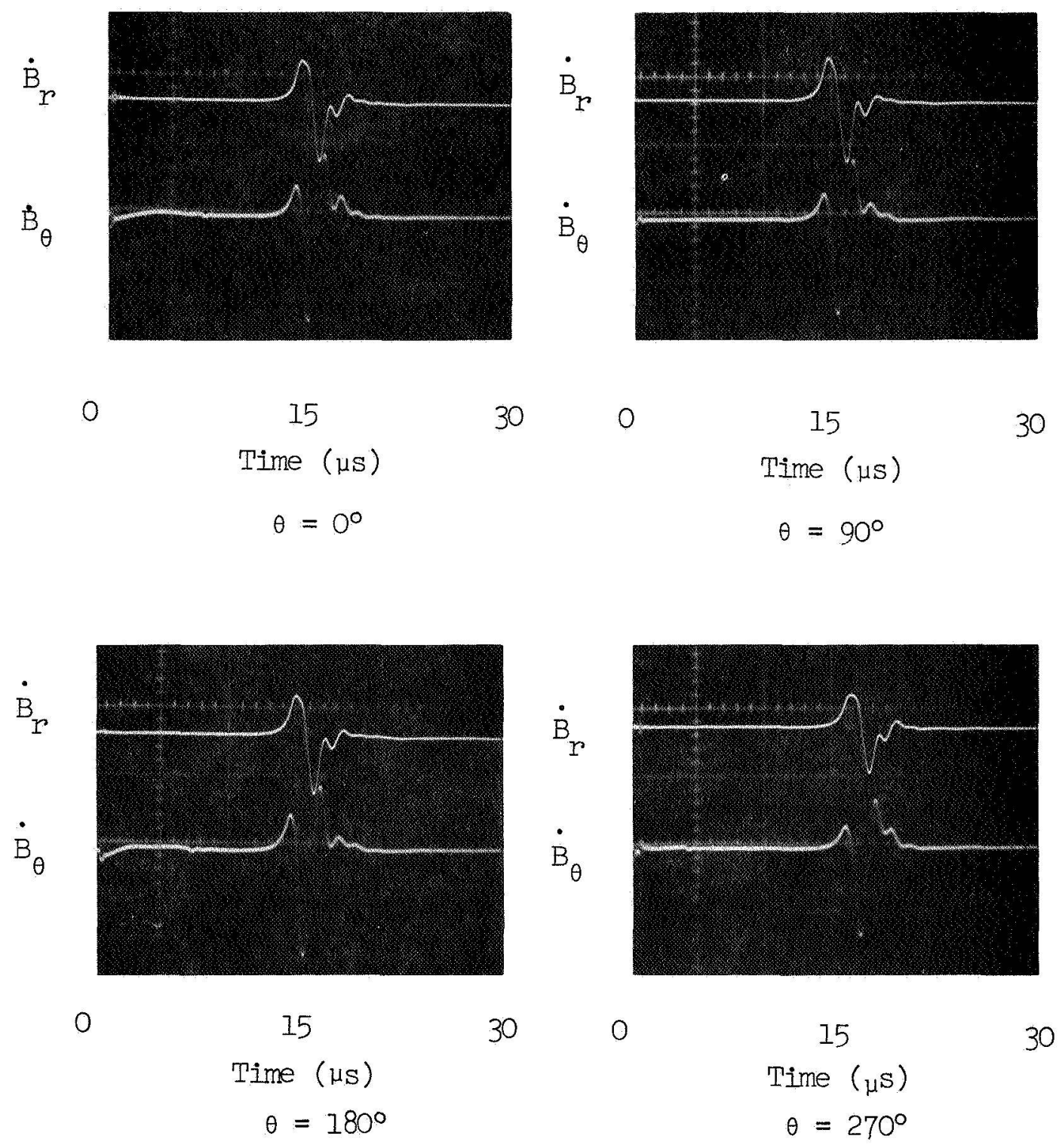

Fig. 5.3 The Variation of $\dot{B}_{r}$ and $\dot{B}_{\theta}$ with the Azimuthal Angle $\theta$ Showing the Symmetry About this Axis 


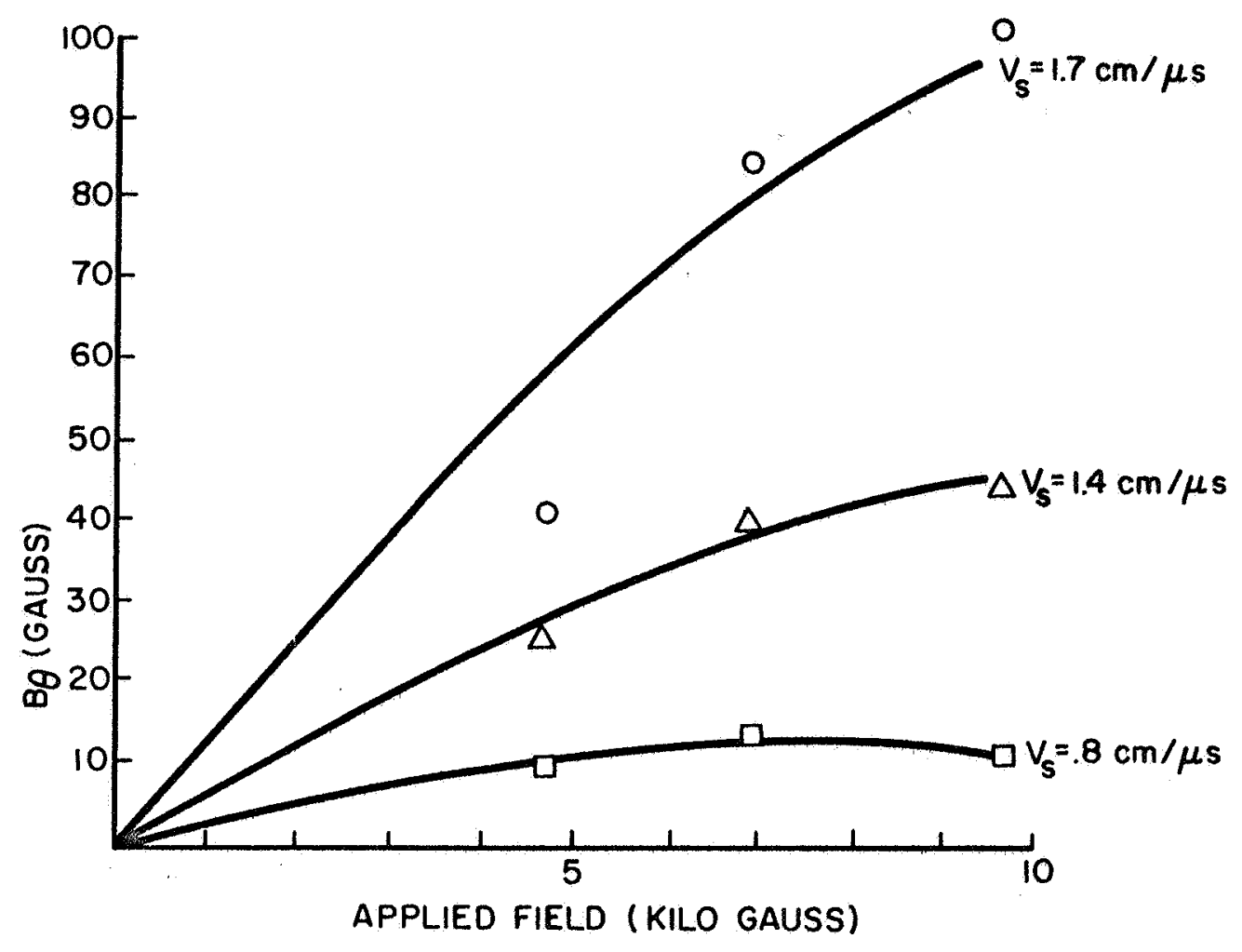

Fig. 5.4 Variation of $\mathrm{B}_{\theta}$ as a Function of the Applied Field $\mathrm{B}_{\mathrm{a}}$ For Various Shock Velocities. (Ambient Pressure$.5 \mathrm{~mm}$ )

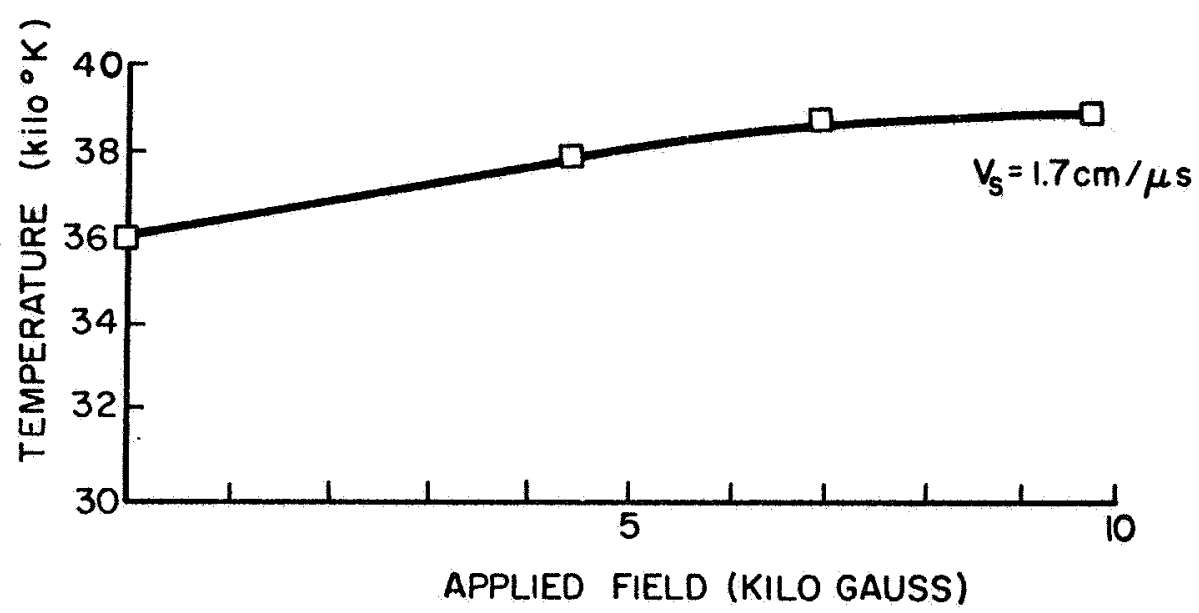

Fig. 5.5 The Variation of Temperature Behind the Shock as a Function of the Applied Magnetic Field. (Ambient Pressure - .5mm). 
dependent variable. This is not too surprising since the plasma conductivity, $\sigma$, is rather insensitive to electron density. This may be seen from Eq. 3.5, in which the electron density only enters in the logarithmic term. Thus, as long as the temperature variations are not large, the conductivity is relatively unchanged and one would expect the MED effects to be similar. However, if the electron density drops to very low value such that the electron-neutral and not the electron-ion collision frequency is dominant, then the conductivity decreases rapidly. It has been found experimentally, as mentioned above, that the MHD effects do not vary appreciably with electron density and fractional ionization. However, if the gas is not preionized the above MHD effects disappear completely. Thus in order to see changes in the magnetic field, it was found that both an axial field and a preionized gas were necessary. This result suggests that these currents have an important role in the structure of the shock front, since if the currents were only important behind the shock the preionization would not affect their presence or absence. Thus, if one wishes to observe the shock structure effects which would be produced by shocks propagating under solar or interstellar conditions, the gas must be preionized.

\subsection{Current Configuration in the Shock}

- Figure 5.6 shows a magnetic probe trace of the $B_{\theta}$ and $B_{r}$ components taken simultaneously. The upper trace is $\mathrm{B}_{\theta}$ ( the integrator was recovering from the preionization during the first $15 \mu \mathrm{s})$ and the lower is $B_{r}$. The disturbance at $37 \mu \mathrm{s}$ is the 


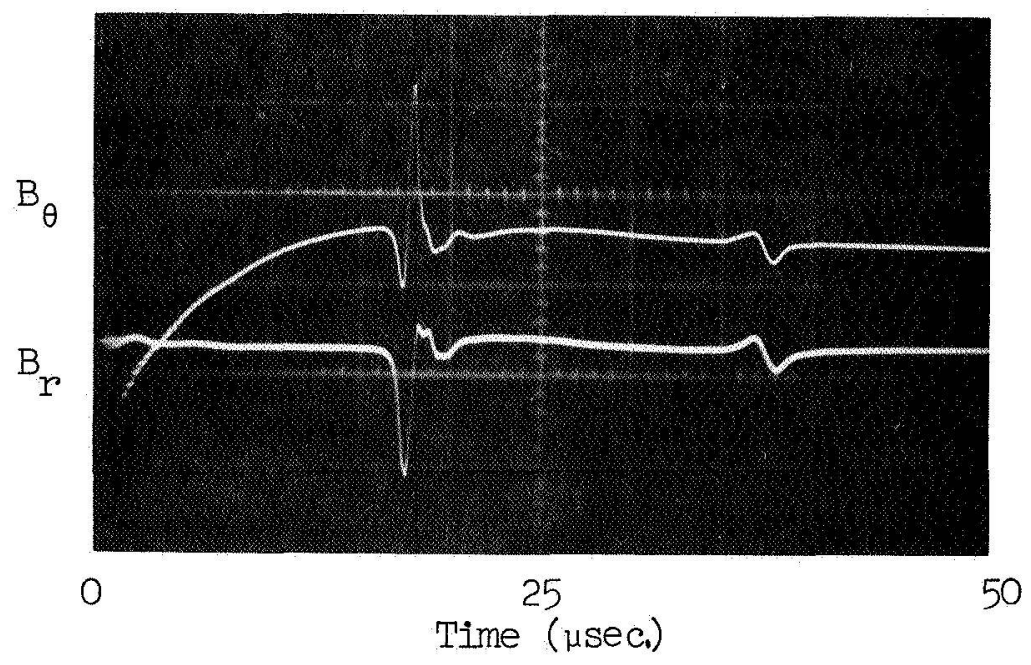

Fig. $5.6 a$

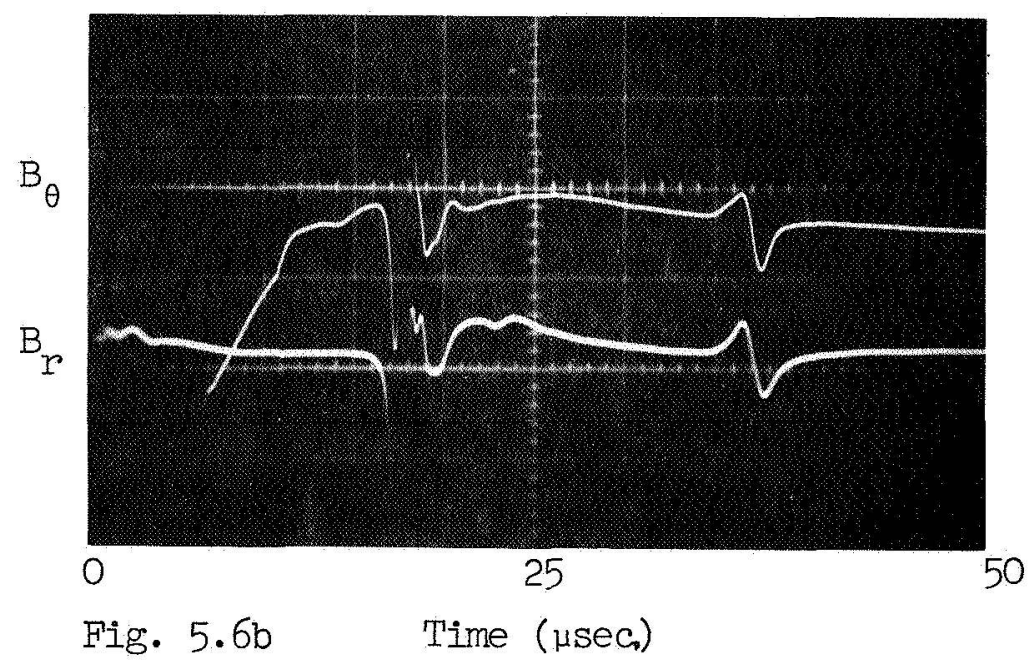

Fig. 5.6 Azimuthal $\left(\mathrm{B}_{\theta}\right)$ and Radial $\left(\mathrm{B}_{\mathrm{r}}\right)$ Jump Fields. During the early stages the $\mathrm{B}_{\theta}$ integrator is recovering from the disturbance due to preionization. The disturbance at $35 \mu \mathrm{sec}$ is the reflected shock. Fig. $4.7 \mathrm{~b}$ is similar to (a) except that the ordinate scale was expanded. 
reflection of the shock off the metallic probe holder. In order to devise a current scheme consistent with the magnetic field measurements, consider the resultant magnetic field if the currents are imagined to flow on the surface of the doughnut shown in cross section in Fig. 5.7, where the axis of doughnut is along the applied magnetic field. The current actually is not imagined to be sharply confined to one doughnut as shown but spread out uniformly in shells so that the magnetic jumps produced will be qualitatively like that in Fig. 5.7. If in addition there is an azimuthal current, like a solenoidal current, then a double humped radial field is produced as shown in Fig. 5.8. This current is responsible also for the magnetic field jump in the longitudinal direction. The magnetic probe measurements in the longitudinal direction show that there is one azimuthal current ring. Therefore in examining Fig. 5.6 a reasonable interpretation would be as follows: (I) the $B_{\theta}$ trace shows a main current loop, preceeded and followed by weaker loops. (2) The $\mathrm{B}_{\mathrm{r}}$ trace shows one large azimuthal loop, rather spread out (as indicated by the long tail) over a distance of about $15 \mathrm{~cm}$. These currents are shown in Fig. 5.9. Fig. 5.10 shows the jump fields for a stronger shock. Some of the details have changed and there is much evidence of field oscillations.

This analysis could probably be carried much further but due to the qualitative nature of the discussion this would not be worthwhile. The important features of the preceeding discussion are first the existence and complete stability (times observed here are at 

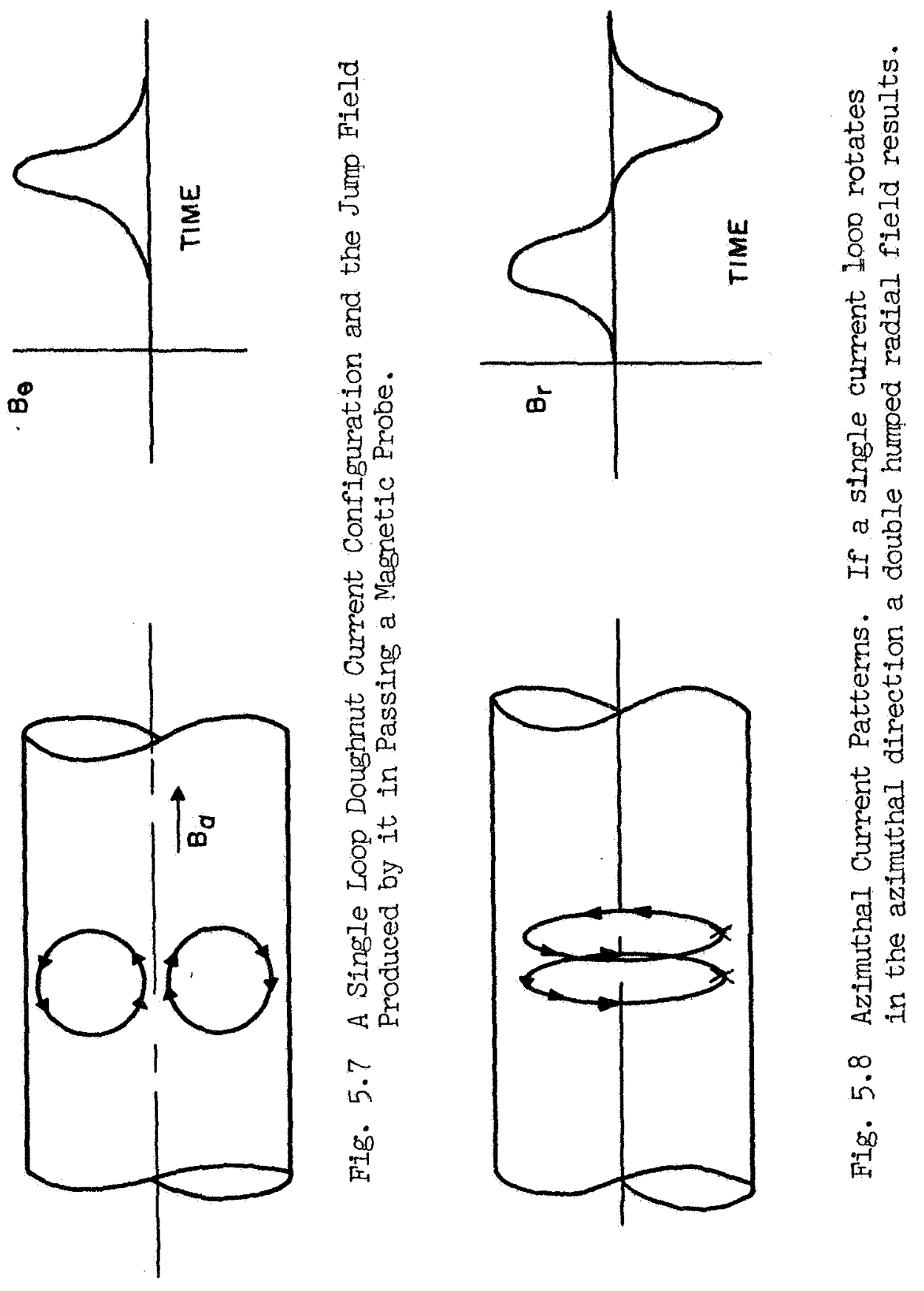

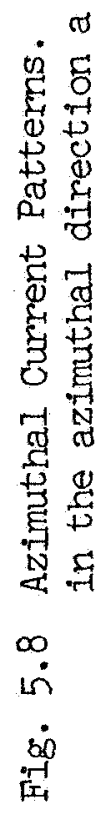




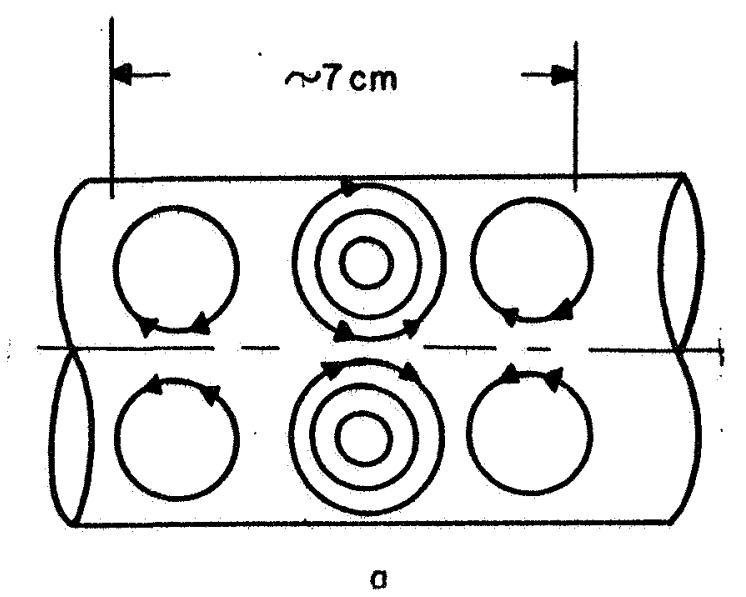

F1g. 5.9a

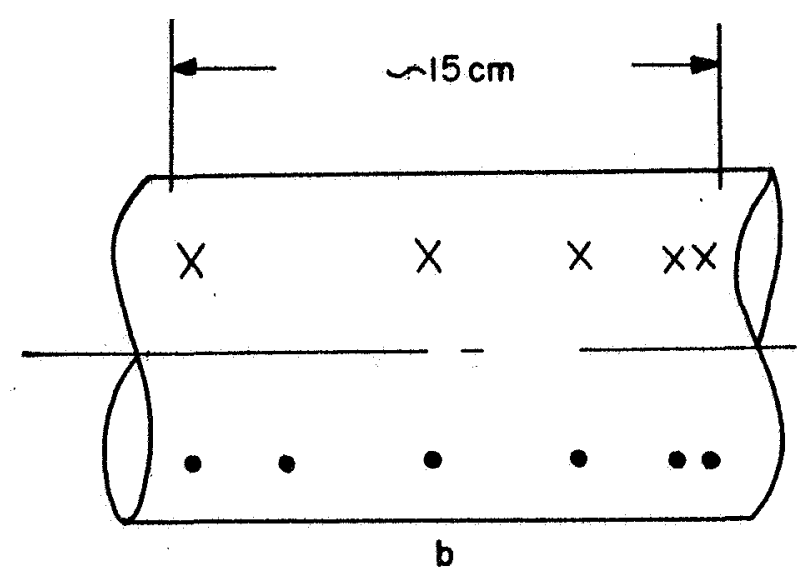

F1g. $5.9 b$

F1g. 5.9 Schematic of Possible Shock Currents Due to the Doughnut Currents (a) and Azimuthal Currents (b), Corresponding to Fig. 5.6 


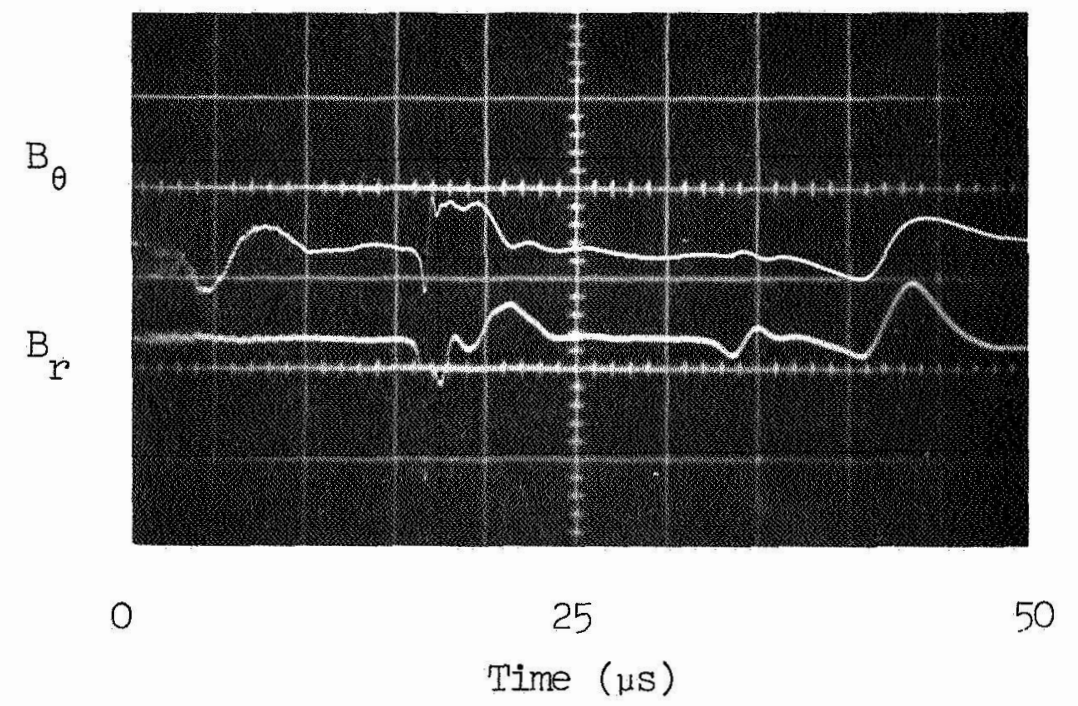

Fig. 5.10 The Azimuthal and Radial Components of the Magnetic Fields Jumps of a Strong Shock 
least 50us after the initiation of the shock wave)of currents in the shock, and second, the existence of separate current loops in the shock (these could be due to the ringing of the shock condenser bank).

\subsection{Summary}

The inclusion of an applied magnetic field produced MHD effects, but qualitative comparison can not be made to MHD planar theory because the shock disturbance was not planar but possessed complete azimuthal symmetry. However, some important results are:

1. The absence of these effects if there is no preionization.

2. The MHD effects are not sensitive to ionization in the range covered by this experiment.

3. The existence of rapid magnetic field oscillations behind the main field jumps. An energy dissipation mechanism for the shock is indicated, because these oscillations eventualiy are damped out.

4. The complete stability of these currents, as long as the shock (or wave) exists. 
CHAPTER VI

\section{GASDYNAMIC SHOCK WAVES}

\subsection{Introduction}

This chapter is concerned with the experimental results where the axial magnetic field was not applied, i.e., the experimental procedure was that the gas was first preionized and then the shock initiating condenser bank was discharged. The shock waves which are produced are termed gasdynamic, or electrogasdynamic, because a charge separation forms within the shock front producing an electric field there. Here, when these designations are employed, it is understood that the gas is preionized to the extent that the electron-ion collision frequency is much greater than the electronatom collision frequency. This is always achieved in this experiment

because the fractional ionization is never less than $\approx 10 \%$. The material in this section falls into two categories: (1) Studies of the shock structure, and in particular the width of the electron thermal layer of the shock (2) Measurements of the temperature behind the shocks as a function of velocity and the comparison of these results to the case studied by other workers where the gas is not initially ionized.

\subsection{Shock Thickness}

The problem of the structure of a gasdynamic shock wave has been treated by a number of workers, but reference here will be made 
only to the treatment of Jaffrin and Probstein. ${ }^{6}$ Their theoretical results agree well with the earlier studies by Jukes ${ }^{7}$ and Shafranov ${ }^{8}$, and the later results of $\mathrm{Jaffrin}^{9}$, but the treatment by Jaffrin and Probstein is more applicable to this work. Their treatment involves the use of the Navier-Stokes equations for electron and ion fluids together with Poisson's equation for the self-induced electric field, assuming that the gas is always completely (and singly) ionized. Thus the heat conductivity and transfer, as well as the electric force, is taken into account. The solution of these equations yields characteristic lengths corresponding to three separate regions in the shock structure. When the shock speed is less than Mach 1.12, there is no abrupt change in the free stream ion and electron velocity and the width of the "shock" is in the order of $\varepsilon \ell_{2}$, where $\varepsilon$ is the mass ratio of the ion to electron ( $=86$ for $\mathrm{He}$ ) and $\ell_{2}$ is the downstream ion-ion mean free path. For stronger shocks there is still no abrupt change in the free stream electron velocity, but the free stream ion velocity changes rapidly over a region of width $\ell_{2}$. Thus there is an ion shock imbedded in a wide electron thermal layer. This latter case is shown in Fig. 6.1, for a Mach 2 shock wave (in the frame of reference moving with the shock) which is taken from Jaffrin and Probstein, where $\sigma$ and $\tau$ refer to the electron and ion temperature and $\omega$ is the ion velocity, all of which are normalized to their final downstream values. The important features are: ( 1 ) in the first layer, of width $\varepsilon_{1} l_{2}$ (or more accurately $2.5 \varepsilon \ell_{2}$, as may be estimated from Fig. 6.1), a marked 


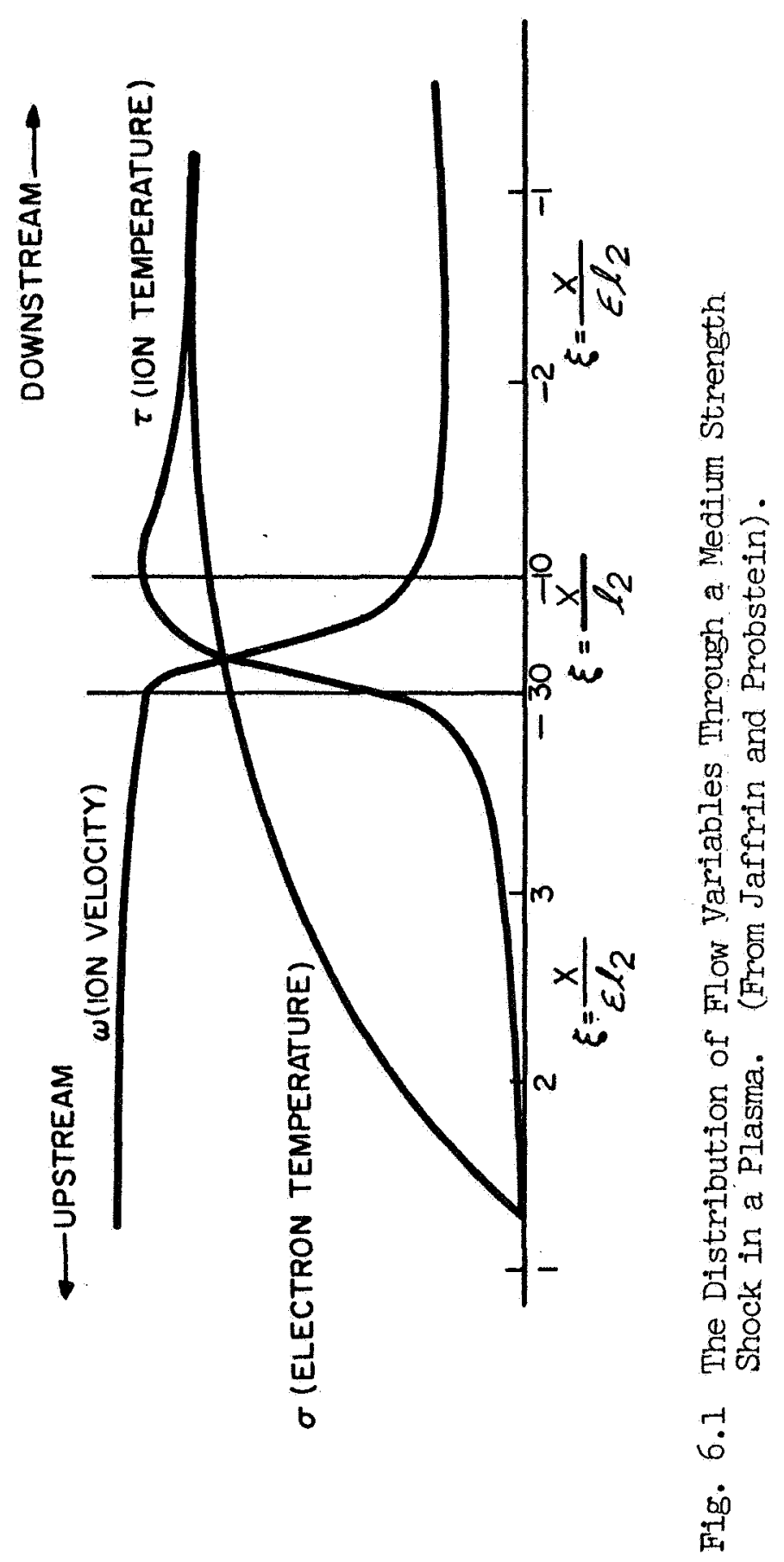


gradual increase in electron temperature reaching nearly its final value at the end of the first layer. The ion velocity and temperature show only slight changes over this region. The fact that the ion velocity does not change appreciably implies that in this region the density changes are also small. (2) In the second layer, of width, $\ell_{2}$, the electron temperature is roughly constant but there is a gross rise in ion temperature and decrease in ion velocity. Since essentially all the ion velocity change takes place in the second layer, this is the region in which the density jumps occur. (3) In the third region, of width $\varepsilon l_{2}$, gross changes having occurred in the first two layers, all variables settle down to their downstream quiescent values. An interesting feature of this region is the overshoot of the ion temperature above the electron temperature. (4) For very strong shocks a precursor electric shock layer appears upstream at the beginning of the thermal layer.

The width of these layers is determined by the downstream ionion mean free path, which is a function of electron density and temperature. Fig. 6.2 shows the parameter, $86 \ell_{2}$. For example, at $10^{16}$ electrons $/ \mathrm{cm}^{3}$ and a temperature of $2.5 \mathrm{ev}$, the width of the electron thermal layer is $2.5 \mathrm{~mm}$ while the ion shock has a width of $2.10^{-2} \mathrm{~mm}$. Since a typical shock velocity is in the order of $2 \mathrm{~cm} / \mu \mathrm{sec}$. this corresponds to an "observation" time of .Iusec. and $I_{\mu s e c}$ respectively. Thus one could not measure the ion shock, even if it were not obscured by the curvature of the shock front, because 


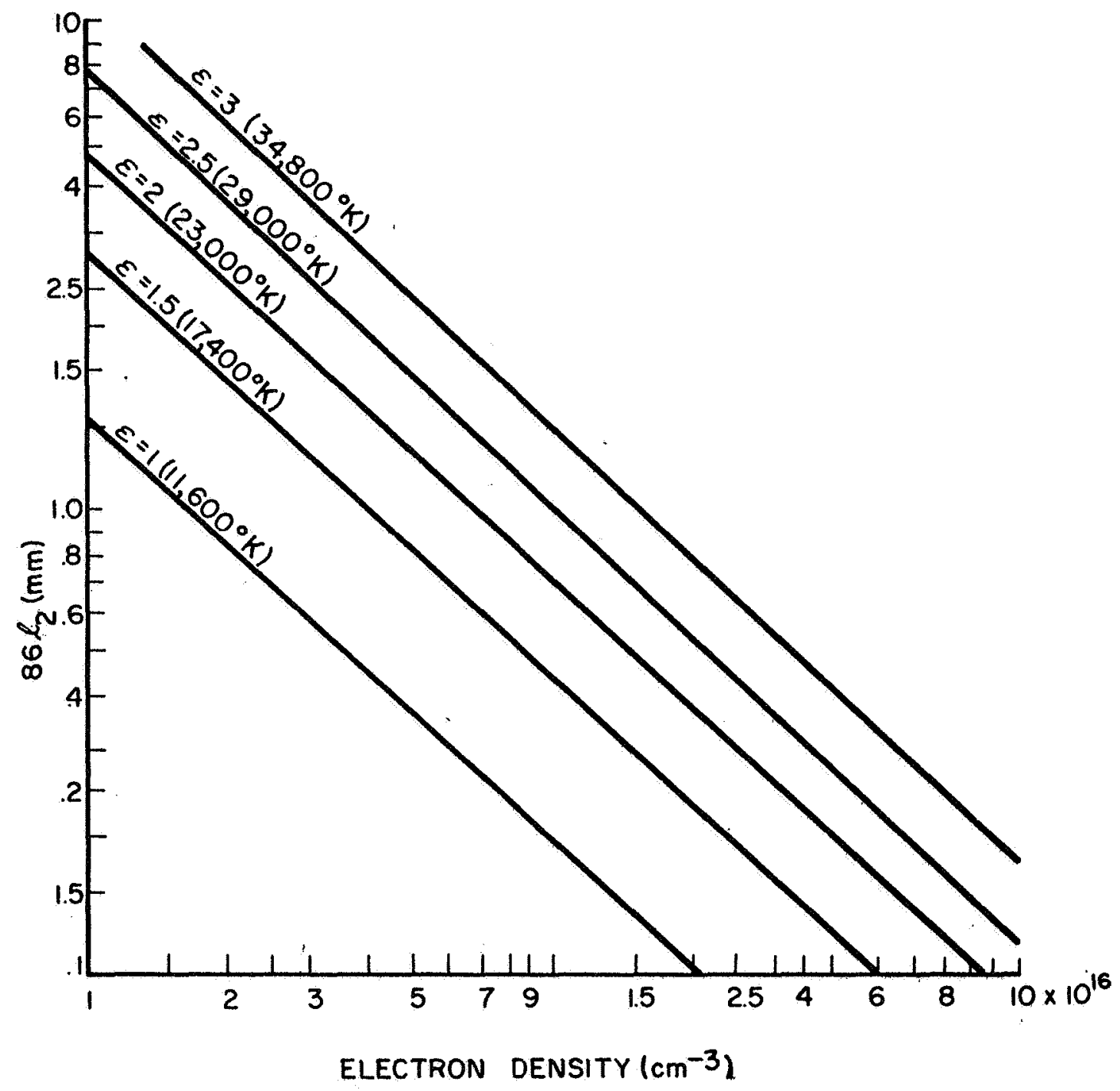

Fig. 6.2 Approximate Width of the Electron Thermal Layer as a. Function of Electron Density and Temperature (in e v.I 
of the difficulty of measuring nano-second events. The thermal layer, on the other hand, is wide enough so that it is a .I to Ius event which may be measured, and further it is clearly marked, at its inception by an increase in electron temperature and at its termination by the abrupt density jump of the ion shock. In this case, as long as the shock curvature is not severe the thermal layer should be observed. (Pictures taken with an STL image convertier camera showed that the curvature was in the order of 10 to 20 percent of the shock tube diameter).

Fig. $6.3 \mathrm{a}$ shows the atom intensity and continuum intensity for a medium strength shock. Fig. $6.3 \mathrm{~b}$ is an expanded portion of Fig. 6.3a showing clearly, by the quick dip in atom radiation, the existence of the first (thermal) layer. The fact that the increase in the continuum intensity during this time is modest, typically only a factor of 2 , is important. This indicates that the electron density (and hence also the ion density) increases by about a factor of $\sqrt{2}$. Thus, there is only a small density change across the first layer, the gross changes occurring in the second layer as predicted by the theory. The electron temperature is indicated by a measure of the ratio of the line to continuum intensity, as discussed in Chapter III. Since the line to continuum ratio is considerably decreased, this indicates a strong rise in the electron temperature in the first layer. It is important to remember that this method of temperature measurement indicates the electron temperature and is insensitive to either the ion temperature or the kinetic atom 


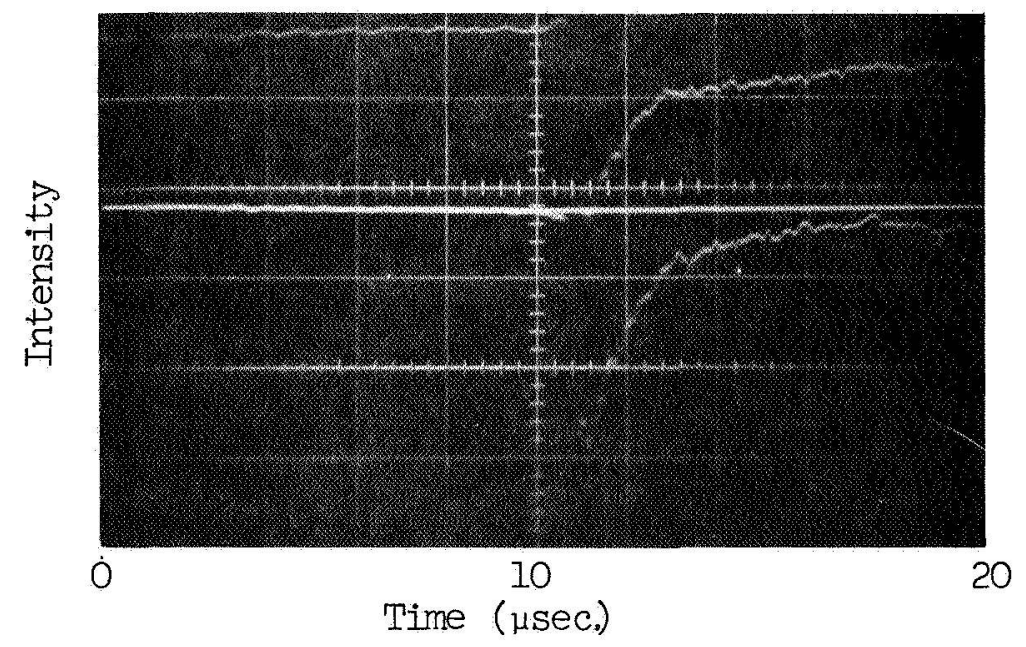

Fig. 6.3a

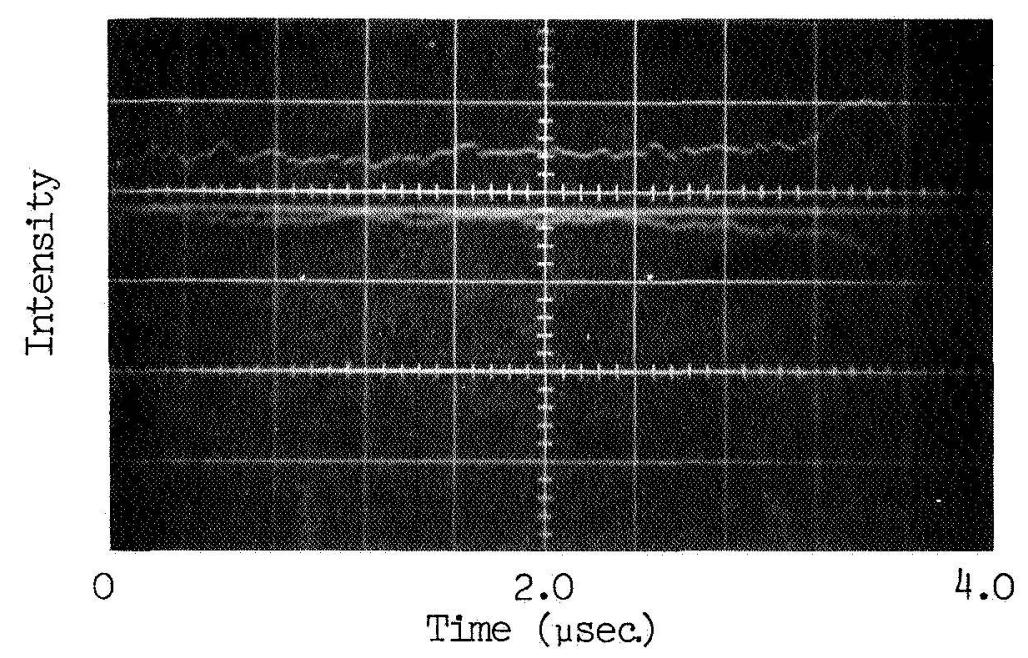

Fig. $6.3 \mathrm{~b}$

Fig. 6.3 Atom (Upper Trace) and Continuum (Lower Trace) Intensities for a Medium Strength Shock $\left(\mathrm{V}_{\mathrm{S}}=1.4 \mathrm{~cm} / \mu \mathrm{sec}\right.$., $\mathrm{N}_{e}$ Behind the Shock $-1.4 \times 10^{16} / \mathrm{cm}^{3}$, Temperature Behind the Shock $-35,000^{\circ} \mathrm{K}$ ). The scales in Fig. $6.3 \mathrm{~b}$ have been expanded to reveal the details of Fig. 6.3a 
temperature. One other possible cause for the dip could be the absorption, by the cooler neutral atoms in the thermal layer, of radiation emitted by the shock, i.e., for example a photon, characteristic of the $4^{3} \mathrm{~F}$ to $3^{3} \mathrm{D}$ level, could be absorbed by an atom in the $3^{3} \mathrm{D}$ state, causing a depopulation of this state. However, as calculated in Appendix 2, the optical depth in the region ahead of the shock is at least $20 \mathrm{~cm}$, so that heating by this method seems unlikely. Fig. 6.4 shows the thickness of the first thermal layer as a function of the electron density behind the shock. The solid and broken lines represent, respectively, the experimental and the theoretical thicknesses based on the experimental electron density and temperature. From Fig. 6.4, it may be seen that the experimental results are about three times too large. Another method of comparing results to theory is to use the measured electron density ahead of the shock to determine $l_{1}$, the ion-ion mean free path ahead of the shock. Then, if the Mach number is known, $l_{2}$ may be determined graphically from the theory of Jaffrin and Probstein. 6 Fig. 6.5 shows such a plot where the solid lines mepresent the theoretical curves for three Mach numbers, plotted as a function of electron density ahead of the shock. The Mach number is difficult to obtain experimentally but is about 2 to 3. Thus, as seen from Fig. 6.5, the experimental agreement is better using this method of comparison.

It is felt that these experimental results are in good agreement with the theory if consideration is taken of the fact that 


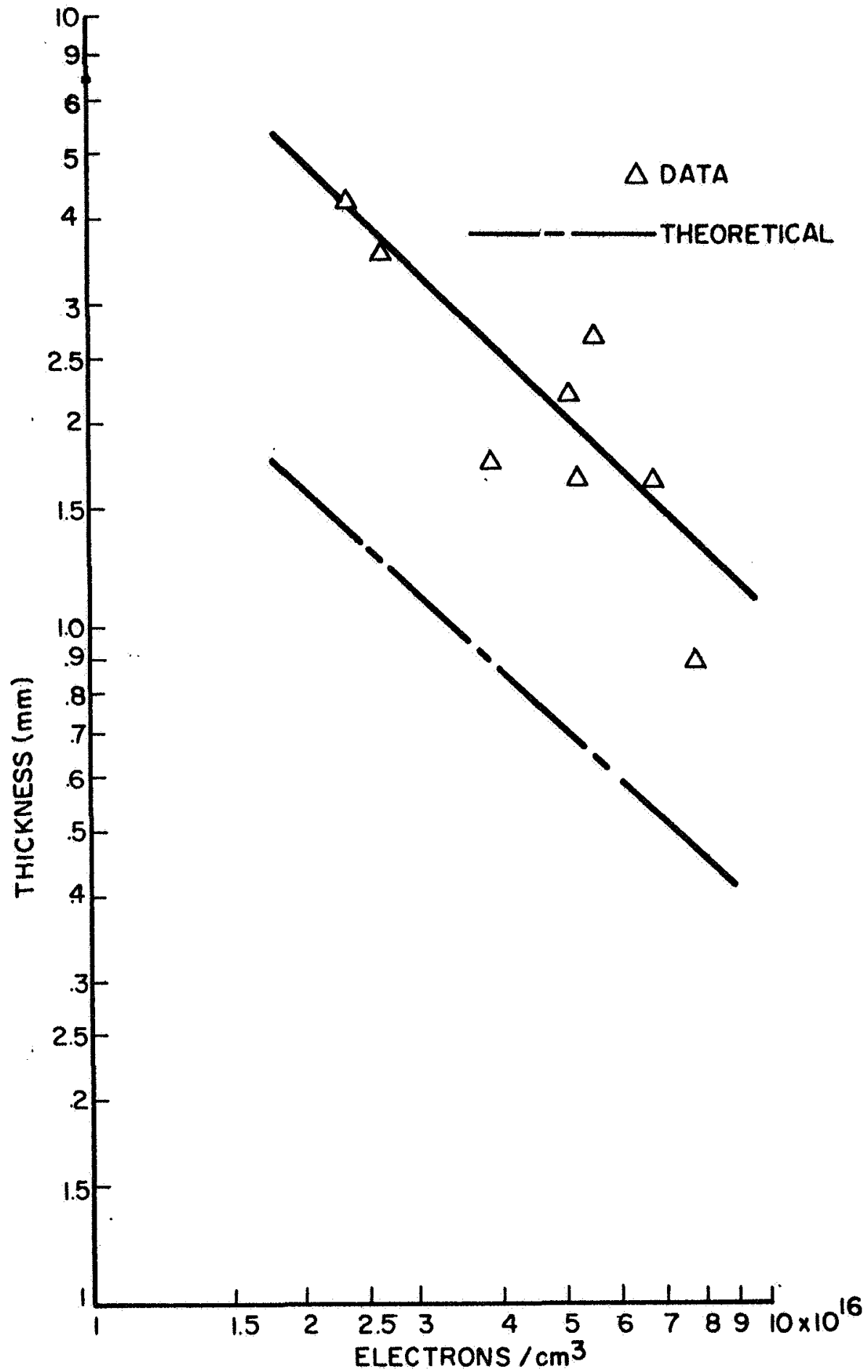

Fig. 6.4 Shock Thickness of the First Layer as a Function of Electron Density Behind the Shock 
53

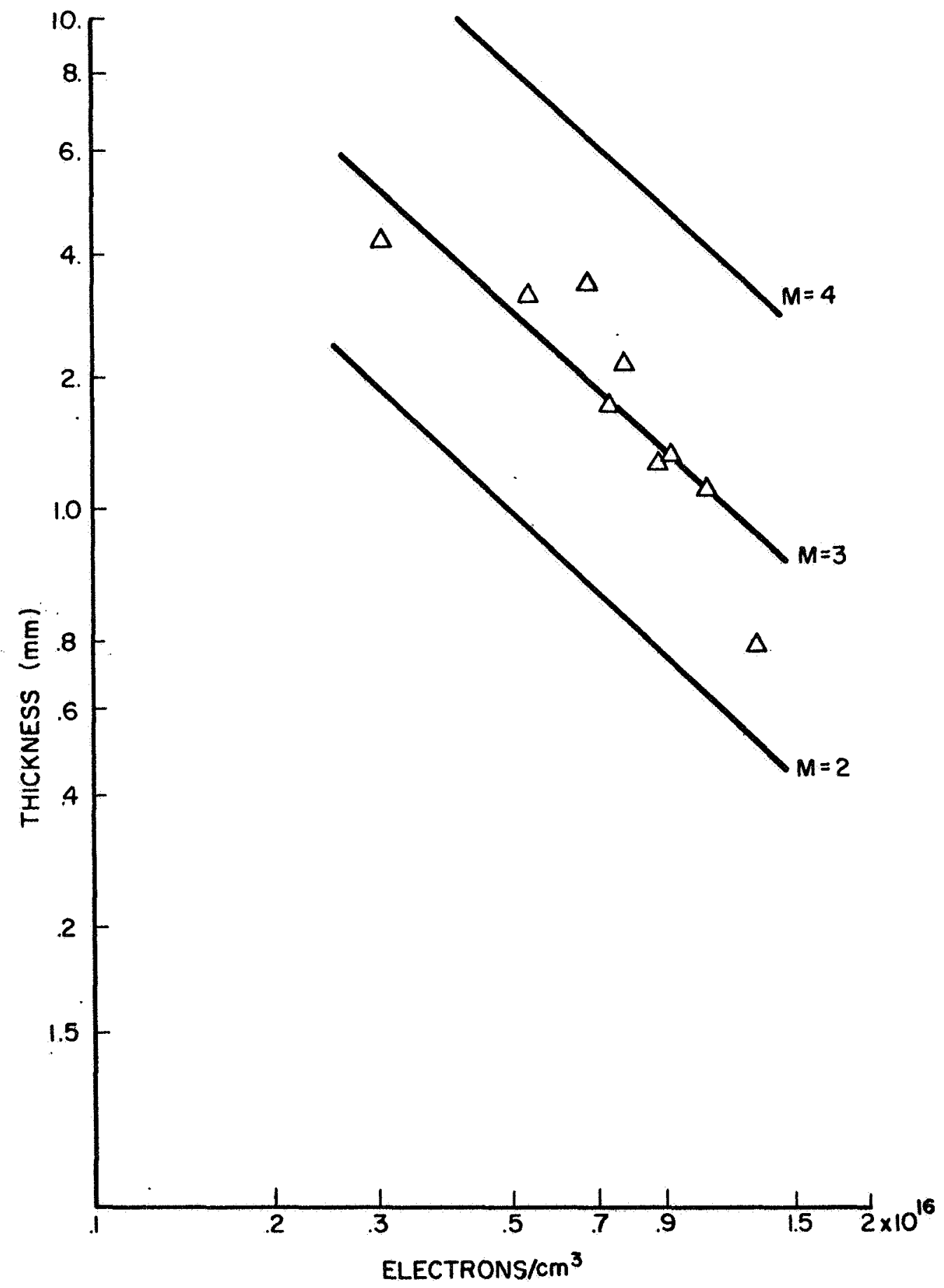

Fig. 6.5 Shock Thickness of the First Layer as a Function of the Electron Density Ahead of the Shock 
the theory assumes a fully ionized plasma, whereas in practice, the experiment was performed with preionizations of from 10 to 50 percent. In view of the statements in Chapter III where preionizations of $85 \%$. were obtained, this may seem contradictory. However, in order to obtain a width large enough to observe, the preionized electron density must be lowered to a value of $25 \times 10^{15}$ per $\mathrm{cm}^{3}$, and a quiescent highly preionized plasma was not obtainable in this region. Thus the shock passes not only though the first ionization, but also in some cases, well into the second ionization stage. The effects of ionization are so important that it is not worthwhile to discuss the merits of the two methods of comparing theory to experiment. (considering just the energetics of the situation, the energy stored in internal ionization energy is greater than either the thermal or directed energy). The fact that one method results in too low an estimate while the other is approximately correct, is very likely an outcome of ignoring the effects of ionization through the shock.

The observance of the first layer, and the good agreement to the theory of Jaffrin and Probstein seems to justify their model, i.e., of a two fluid approach using the Navier-Stokes equations together with Poisson's equation, at least as far as a quantitative description of the first layer is concerned. Further, it confirms the basic correctness of quantities involved in these equations, such as coefficients of shear viscosity and thermal conductivity, collision mean free paths, etc. 


\subsection{The Sound Speed in a PLasma}

Fig. 6.6 shows the experimental shock speed as a function of the energy of the capacitor bank, when the quiescent gas ahead of the shock disturbance was $80 \%$ singly ionized at a temperature of $22,000^{\circ} \mathrm{K}$. Notice that the velocity is nearly linear with energy, the last measured data point being at $.7 \mathrm{~cm} / \mu \mathrm{s}$. Presumably then, the sound speed must be no greater than $: 7 \mathrm{~cm} / \mu \mathrm{s}$. The sound speed in a fully ionized plasma may be computed, assuming an ideal gas, from the following expression:

$$
\begin{aligned}
c & =\sqrt{\frac{\gamma P}{\rho}}=\sqrt{\frac{\gamma\left[N_{e}+N_{a}+N_{i}\right] k T}{\rho_{e}+\rho_{a}+\rho_{i}}} \\
& \cong \sqrt{\frac{2 \gamma k T}{M}}
\end{aligned}
$$

where

$c=$ the sound speed

$P=$ pressure of ambient gas

$\rho=$ total mass density of ambient gas

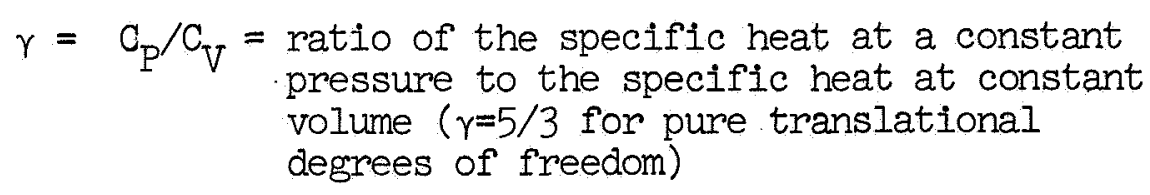

$$
\begin{aligned}
& \mathrm{N}_{e}, \mathrm{~N}_{\mathrm{a}}, \mathrm{N}_{\mathrm{i}}= \begin{array}{l}
\text { ambient number density per unit volume of } \\
\text { electrons, atoms, and ions }
\end{array} \\
& \rho_{\mathrm{e}} \rho_{\mathrm{a}} \rho_{\mathrm{i}}=\text { ambient mass density of electrons, atoms, and ions } \\
& \mathrm{K}=\text { Boltzman's constant } \\
& \mathrm{T}=\text { temperature, }{ }^{\circ} \mathrm{K} \\
& \mathrm{M}=\text { mass of He atom. }
\end{aligned}
$$




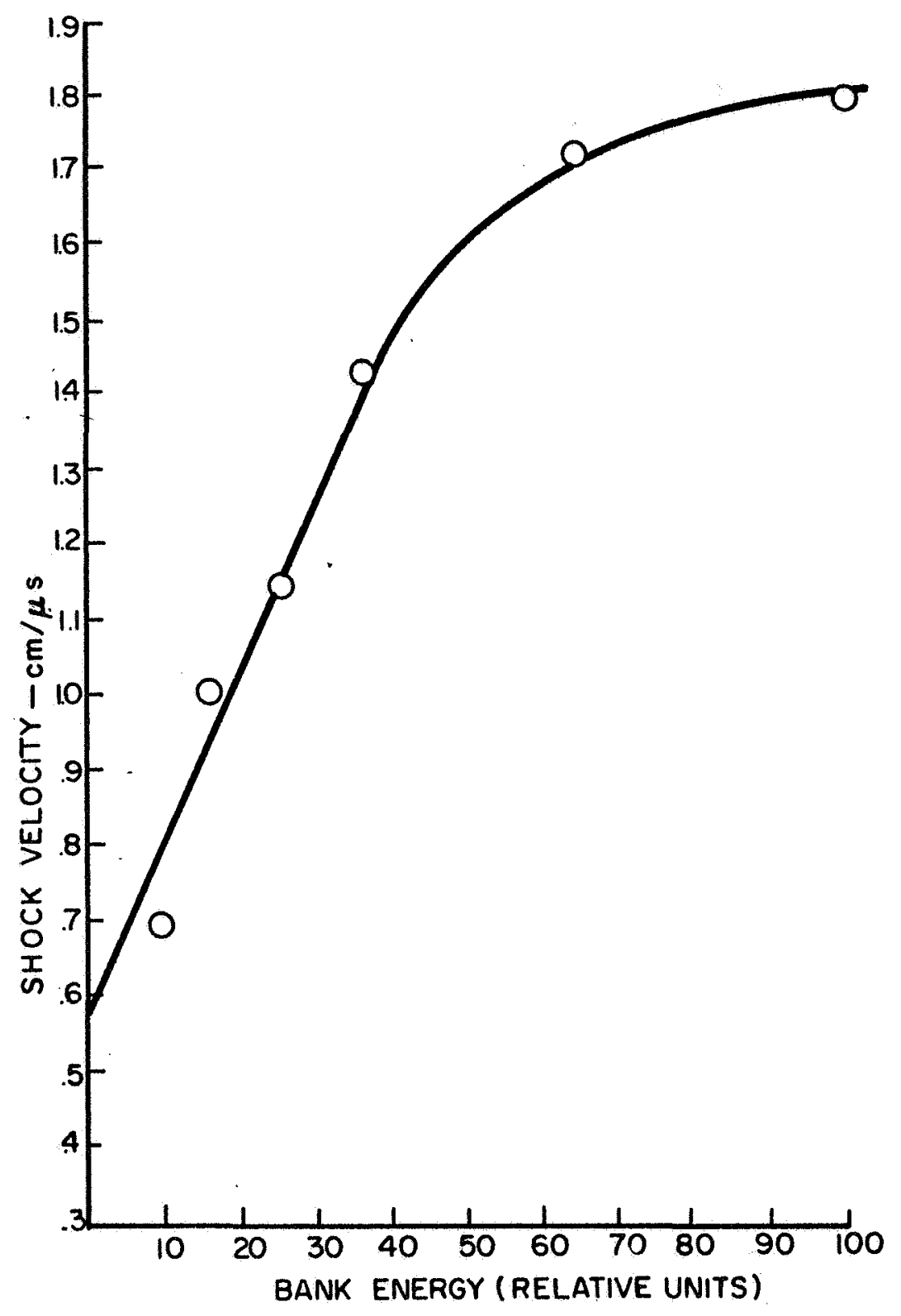

Fig. 6.6 Shock Velocity as a Function of the Energy of the Capacitor Bank in Relative Units. (100 corresponds to 10,000 Volts) 
This equation yields a sound speed of $1.1 \mathrm{~cm} / \mu \mathrm{s}$ using the above temperature $\left(22,000^{\circ} \mathrm{K}\right)$ and $\gamma=5 / 3$. Therefore, the calculated value is too large, the discrepancy not being removed even if possible experimental errors are taken into account. The value of $\gamma$ is open to immediate suspicion, since it is determined from the energy state equation for an ideal gas where no ionization takes place. Assuming an ideal gas, but permitting ionization, the value of $\gamma$ may be determined as a function of temperature. This derivation is carried out in Appendix $\mathrm{C}$, always assuming equilibrium, and a plot of $\gamma(T)$ is shown in Fig.6.7 for the first ionization of helium. The curve is best described as almost being a square well, extending over a range in temperature from .9ev to 2.2ev. In this range $\gamma$ falls to a value of $u l .1$. It is interesting to compare the width of this region to the region in which the fractional ionization is rapidly changing. This also is shown in Fig. 6.7 and it may be seen that $\gamma$ is affected well into that part of the curve where the fractional ionization changes only slightly.

If the value of 1.1 is used for $\gamma$ instead of $5 / 3$, the theoretical sound speed is $0.9 \mathrm{~cm} / \mu \mathrm{s}$, which is still too high, but within the combined experimental errors. If the straight line approximation of Fig. 6.6 is valid down to zero energy, the sound speed in the limit is $.5 \mathrm{~cm} / \mu \mathrm{s}$, which again would be too much of a discrepancy. Unfortunately, velockty measurements below $.7 \mathrm{~cm} / \mu \mathrm{s}$ were difficult so that experimental data is lacking for lower 


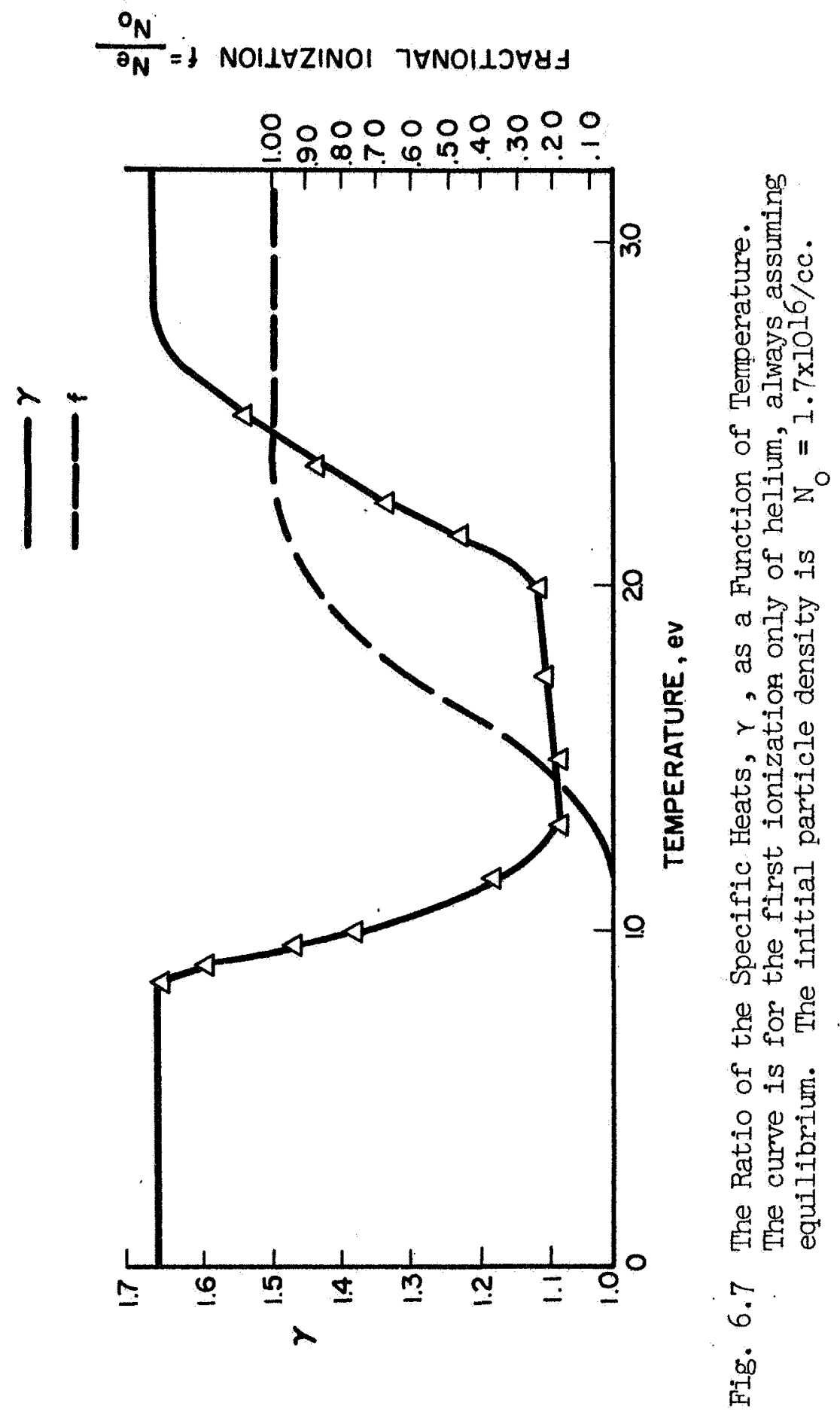


energies.

The importance of the above discussion is that the theoretical sound speed is based on the ideal gas law, which is used as a basis for shock theories. A disagreement between the theoretical and measured speeds would mean that the ideal gas law would not be valid for this case of interacting particles.

\subsection{Shock Structure for an Ionizing Shock Wave}

The results of Section 6.3 have an interesting application to shock waves. If a large disturbance is initiated in a gas, it tends to coalesce and form a shock wave. Imagine the disturbance to be a superposition of linear waves, and then, since the temperature increases through the disturbance, each succeeding wave travels faster $(c \alpha \sqrt{\gamma T})$. This process continues until a shock forms and then the above argument fails. The important point is the fact that rT grows as the temperature increases. The above argument is correct if applied to an ideal gas without ionization.' A qualitative understanding of the effects of ionization may be acquired if, in analogy with the above argument, the sound speed is determined for an ionizing gas. Using Eq. 6.1, $\gamma=\gamma(T), p \cong N_{0} M$, and the fact that $\mathrm{N}_{a}+\mathrm{N}_{e}+\mathrm{N}_{i}=\mathrm{N}_{0}(I+f)$, the sound speed is

$$
c=\frac{\sqrt{(I+f) \gamma(T) k T}}{M} .
$$

That this equation is correct, with the $\gamma(T)$ as determined in Section 6.3 , may be verified by returning to a more basic definition 
of the sound speed, viz., $c=\sqrt{B / p}$ where $B$ is the bulk modulus and $\rho$ is the mass density. A plot of $\frac{c^{2}}{(e / M)}$ determined from Eq. 6.2 is shown in Fig. 6.8, which again is for the first ionization only of helium. The interesting feature is the decrease of sound speed with increasing temperature at the inception of ionization ( $1 \mathrm{ev}$ ). This means that a large amplitude wave (or several large amplitude waves) could split apart rather than coalesce in this temperature range, forming separate disturbances. In this sense the disturbance (and possibly also the shock) is unstable.

Up to this point the effects of the second ionization of helium have been ignated. It is probable that a similar effect in $\gamma$ exists there, but this must be decided by an exact solution considering the simultaneous effect of the first and second ionization stages. This is so because, to the extent that $\gamma$ is affected, the first ionization is by no means complete when the second ionization begins. If this second critica.l point exists, it will occur at a temperature of about $25,000^{\circ} \mathrm{K}$ (an estimate based on the behavior of the first critical point).

The experimental data. shows evidence in some cases of such splitting. Fig. 6.9 shows the atom (upper trace) and continuum (lower trace) intensities showing that the intensity in the front does not rise smoothly but is jagged. Fig. 6.10 shows a strong shock which was observed at a distance of $50 \mathrm{~cm}$ from the "T" so that the shock was considerably weakened when observed. Rather than 


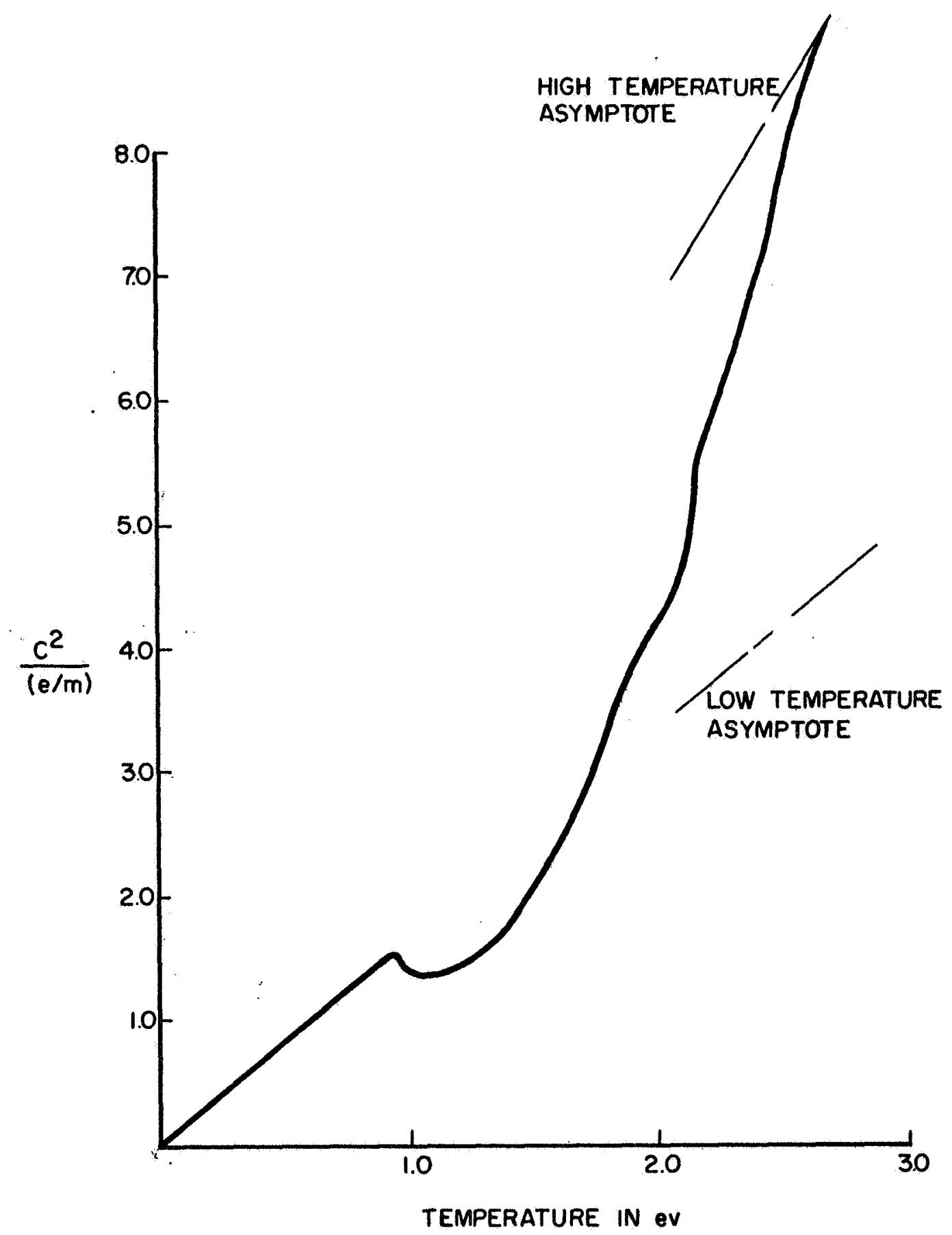

Fig. 6.8 The Square of the Sound Speed (In Units of e/m) as a Function of Temperature in e. $\mathrm{V}$ for the First Ionization of Helium. 


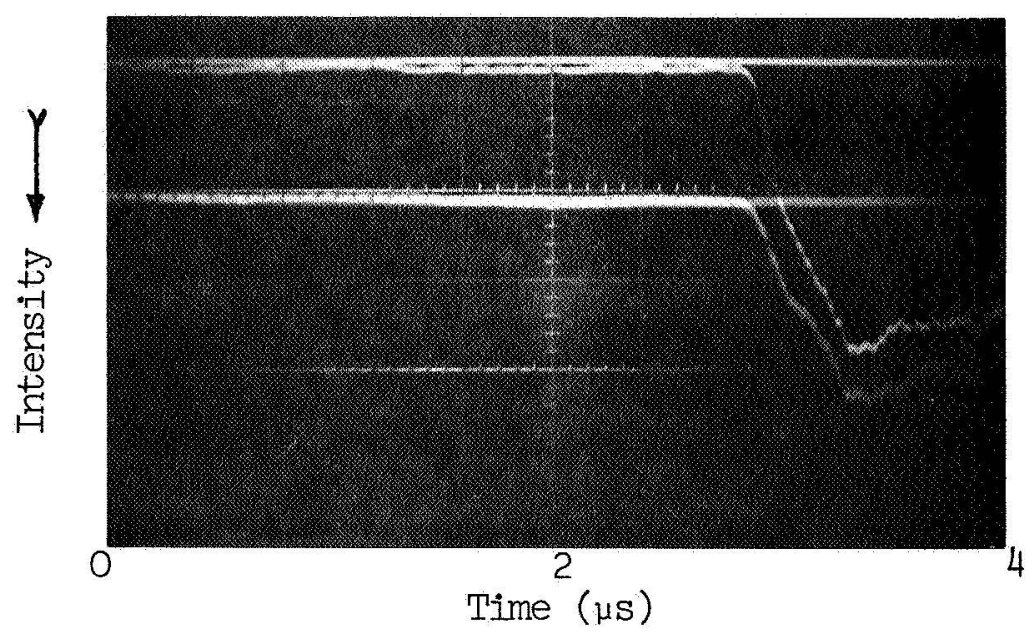

Fig. 6.9 Atom (Upper Trace) and Continuum (Lower Trace) Showing the Irregularity in the Shock Front. Data Taken $20 \mathrm{~cm}$ from the "T"

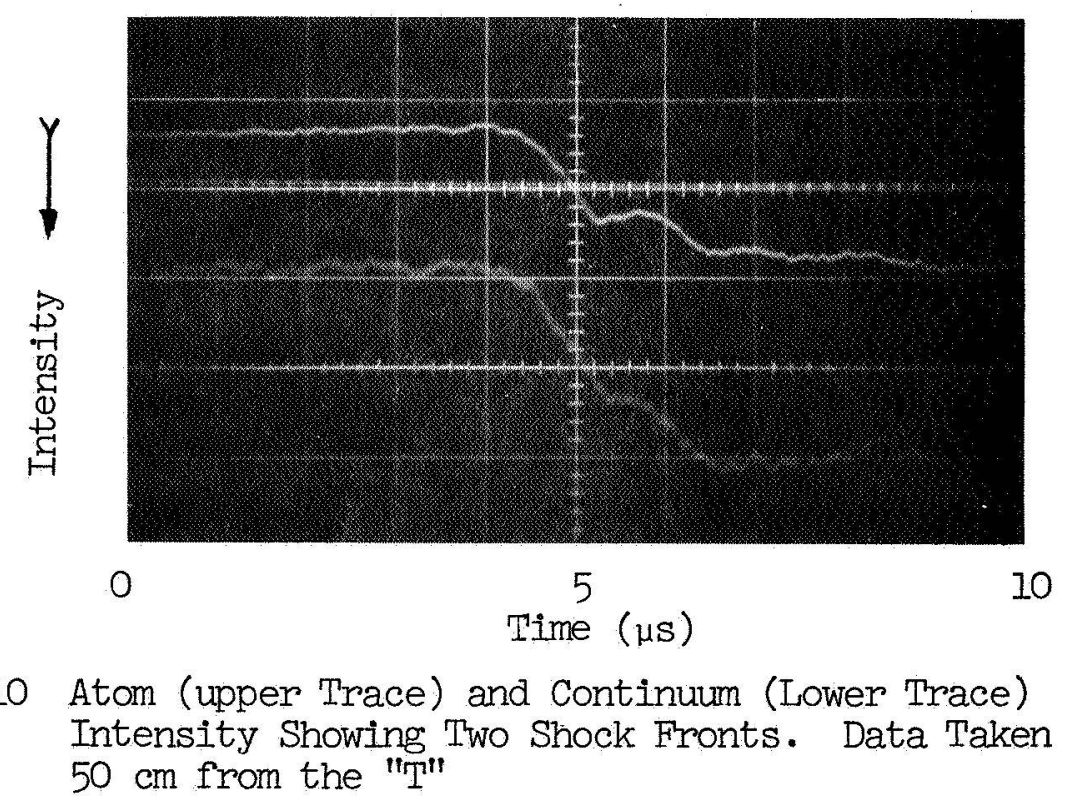


being a smooth shock there is a plateau region in the center of the shock. The temperature in the plateau is measured at $24,000^{\circ} \mathrm{K}$, which is close to the estimated value of the second critical temperature of helium.

In view of the preceeding remarks it seems plausible that, under some circunstances, separate disturbances may be created from a single disturbance, or multiple disturbances would fail to coalesce. This effect would have important consequences on the structure, stability, and formation of the shock and thus should not be ignored in any shock theory.

\subsection{Temperatures Behind the Shock Wave}

As part of an investigation of the properties of the "T" tube, Mc Lean ${ }^{15}$, et al , made temperature measurements behind the shock as a function of shock velocity, for purposes of comparison with the Rankine-Hugoniot equations (there was no intentional preionization in this experiment). It was found in this experiment that the temperatures determined by spectroscopy were too high. Fig. 6.11 shows a comparison of their data and that of the present experiment. Curve (I) represents their computer calculations using the Rankine-Hugoniot equations, where the gas ahead of the shock was assumed to be cold (room temperature). As may be seen, the data of Mc Lean did not agree with the curve. After considering several mechanisms, their conclusion was that the cold gas was being pre- 


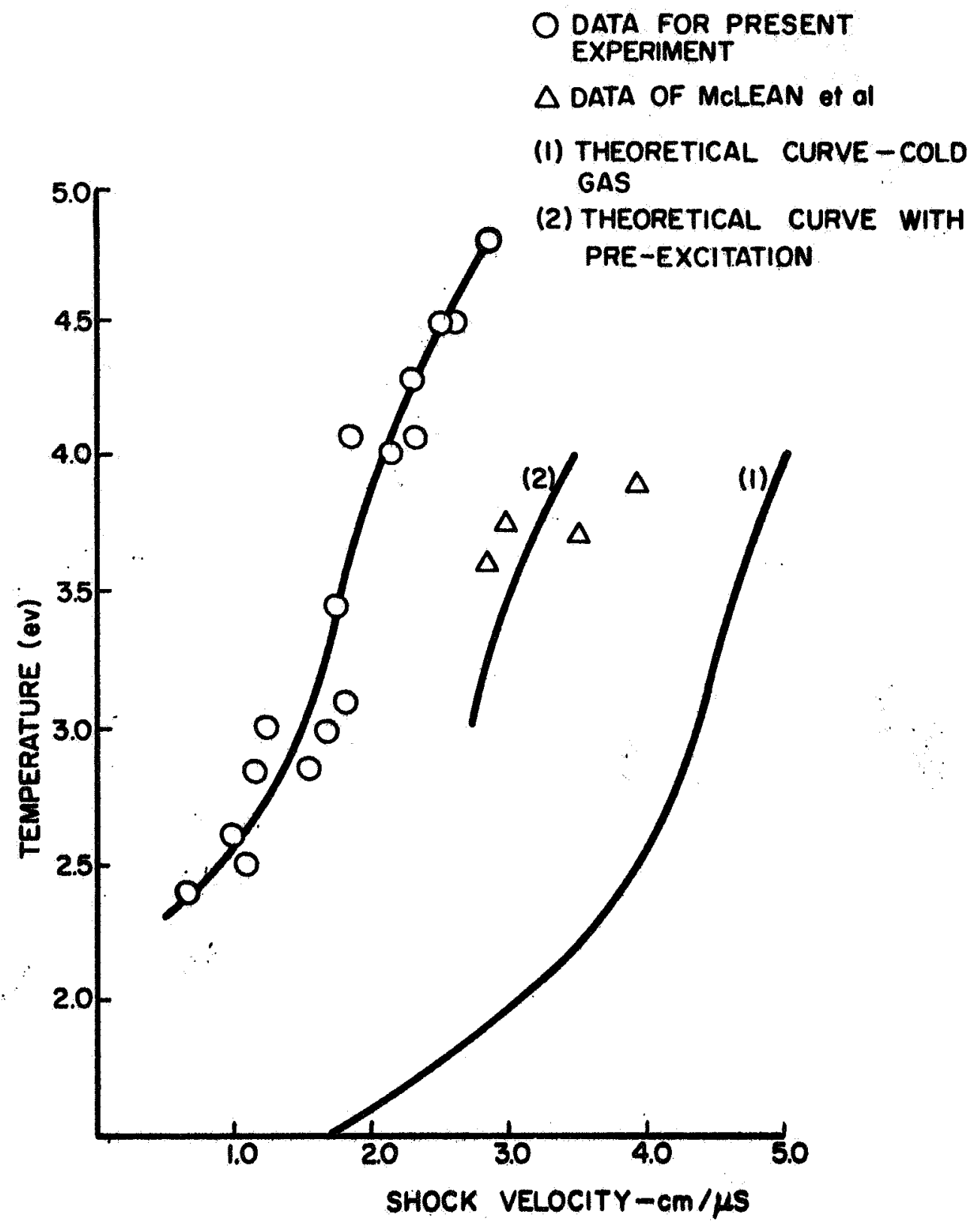

F1g. 6.11 Temperature Behind the Shock as a Function of Shock Velocity 
excited by the ion resonance radiation emitted at the "T" during the first "ring" of the shock condenser bank. Their calculations led them to believe that the pre-excited gas had negligible kinetic energy, but was almost completely pre-excited. On this basis they calculated curve (2) in Fig. 6.11, which agreed well with their data. In the present experiment it was found that the measured temperatures were even higher than those of McLean. If one applies the same sort of logic to the present case, the data is reasonable. The gas is already highly ionized due to the preionization, and is receptive to pre-excitation of the ionic states, since ions are already in existence. The effect on the temperature would indicate that the fraction of pre-excited ions is large.

\subsection{Summary}

Gas dynamic shocks were initiated in highly pre-ionized helium, and the experimental results compared to the shock theory presented by Jaffrin and Probstein. ${ }^{6}$ It was possible to verify the existence of, and quantitatively measure the thickness of the first layer, as predicted by their theory. The experimental results were within

a factor of three of the theoretical predictions in spite of the fact that the theorry was developed only for a fully ionized plasma, whereas in the actual case there was considerable ionization across the shock.

The velocity of weak shock waves was measured, and it was found 
that this velocity was considerably lower than the theoretical sound speed. It was pointed out that this discrepancy could be removed, within experimental error, if ionization effects were taken into account. In addition, it was shown that the ionization effects caused a decrease in sound speed for increasing temperature early in the ionization stage. This could cause a large amplitude wave (or several large amplitude waves) to form multiple disturbances, instead of the ususal single shock wave. Some experimental evidence was presented to support this idea.

The temperature behind the shock wave as a function of shock velocity was measured and compared to those obtained by McLean 15 et al. The temperature behind the shock, in the present case, where the gas was highly preionized, was found to be even higher than those of McLean. It is assumed this is due to an even further enhancement of the preionization, due to absorption by the ions in the plasma of the HeII resonance radiation from the "T" . 
CHAPTER VII

\section{SUMMARY AND COMMENTS}

Gasdynamic and magnetohydrodynamic shock waves were initiated in highly preionized helium. The areas of interest may be broadly classified within three groups; (1) a study in the after-glow period was made of the characteristics of the high current discharge used for preionization (2) MHD effects were observed via provision of a longitudinal magnetic field and (3) a study of gasdynamic shock waves was accomplished.

Spectroscopic methods were used to determine the state of the plasma in the early after-glow following a high current discharge. It was found that the plasma was in local thermal equilibrium and nearly completely ionized. The temperature and electron density during this period was in the order of $22,000^{\circ} \mathrm{K}$ and $3 \times 10^{16}$ electrons/ $\mathrm{cm}^{3}$ respectively. It is felt that further improvements could be made by (1) forcefully interrupting the current discharge at an earlier more appropriate time and (2) increasing the diffusion cooling time by increasing the ambient pressure and using a larger diameter tube. Also, for this method to be firmly established as a method of obtaining a high density plasma, a quantitative timehistory study of the impurities in the early after-glow is required, and an effort made to eliminate these impurities.

The inclusion of an applied magnetic field produced MHD effects, 
but quantitative comparison can not be made to MHD planar theory because the shock disturbance was not planar but possessed complete azimuthal symmetry. However, some important results are:

1. The absence of these effects if there is no preionization.

2. The MHD effects are not sensitive to ionization in the range covered by this experiment.

3. The existence of rapid magnetic field oscillations behind the main field jumps. An energy dissipation mechanism for the shock is indicated, because these oscillations eventually are damped out.

4. The complete stability of these currents, as long as the shock (or wave) exists.

Gasdynamic shocks were initiated in highly preionized helium, and the experimental results compared to the shock theory presented by Jaffrin and Probstein ${ }^{6}$. It was possible to verify the existence of, and quantitatively measure: the thickness of, the first layer as predicted by the theory. The experimental results were within a factor of three of the theoretical predictions in spite of the fact that the theory was developed only for a fully ionized plasma, whereas in the actual case there was considerable ionization across the shock.

The velocity of weak shock waves was measured, and it was found that this velocity was considerably lower than the theoretical sound speed. It was pointed out that this discrepancy could be removed, 
within experimental error, if ionization effects were taken into account. In addition, it was shown that the ionization effects caused a decrease in sound speed for increasing temperature early in the ionization stage. This could cause a large amplitude wave (or several large amplitude waves) to form multiple disturbances, instead of the usual single shock wave. Some experimental evidence was presented to support this idea.

The temperature behind the shock wave as a function of shock velocity was measured and compared to those obtained by McIean ${ }^{15}$ et al. The temperature behind the shock, in the present case, where the gas was highly preionized, was found to be even higher than those of McLean. It is assumed this is due to an even further enhancement of the preionization, due to absorption by the ions in the plasma of the HeII resonance radiation from the "T" .

Some complications were experienced with the use of helium because of the importance of both stages of ionization. It would be desirable to use a gas in which only one stage of ionization would be important, although the choice of this gas is not obvious. 


\section{APPENDIX A}

When the radiation from a plasma is computed, the assumption is that the effective radiating volume is $\mathrm{Al}$, where $\mathrm{A}$ is the area focused into the plasma and $\ell$ is the length of the plasma (see Fig. A-1), even though in general the length of the plasma is large compared to the region over which the system is focused. It can be shown, as follows, that the total intensity is still Al.

Consider Flg. A-I, which shows a uniformly radiating plasma which emits energy, $I_{v}$ /unit volume/unit solid angle. The lens system is assumed to "transform" the area $A$ to the area $A_{S}$, i.e., all rays leaving the volume, $\Delta \mathrm{V}=\mathrm{A} \Delta \mathrm{x}$, at one focus are assumed to pass thru $A_{S}$ and vice versa. The energy at $A_{S}$ due to $\Delta V$ is then $I_{S}=I_{V} A \Delta x \Omega$. The area $A$ is assumed to be macroscoplc, while the volume $d^{\prime}$ is microscopic, so that the contribution to the energy at $A_{S}$ due to $\Delta V^{\prime}$ is

$$
\begin{aligned}
\Delta I_{S}^{\prime} & =\int I_{v} d \Omega^{\prime} d V^{\prime}=\int I_{V}\left(\frac{A}{r^{2}}\right) d V^{\prime}=\frac{I_{V}}{r^{2}} A \int d V^{\prime}=\frac{I_{V} A V^{\prime}}{r^{2}} \\
& =\frac{I_{V}}{r^{2}} A A^{\prime} \Delta x=I_{V^{A}}\left(\frac{A^{\prime}}{r^{2}}\right) \Delta x=I_{V} A \Omega \Delta x \\
& =\Delta I_{S} .
\end{aligned}
$$

Thus the two contributions for equal $\Delta \mathrm{x}$ are equal and $I_{S}=A l$, as long as the end caps are spherical or nearly so. It was assumed above for simplicity that the focus $A$ was within the 


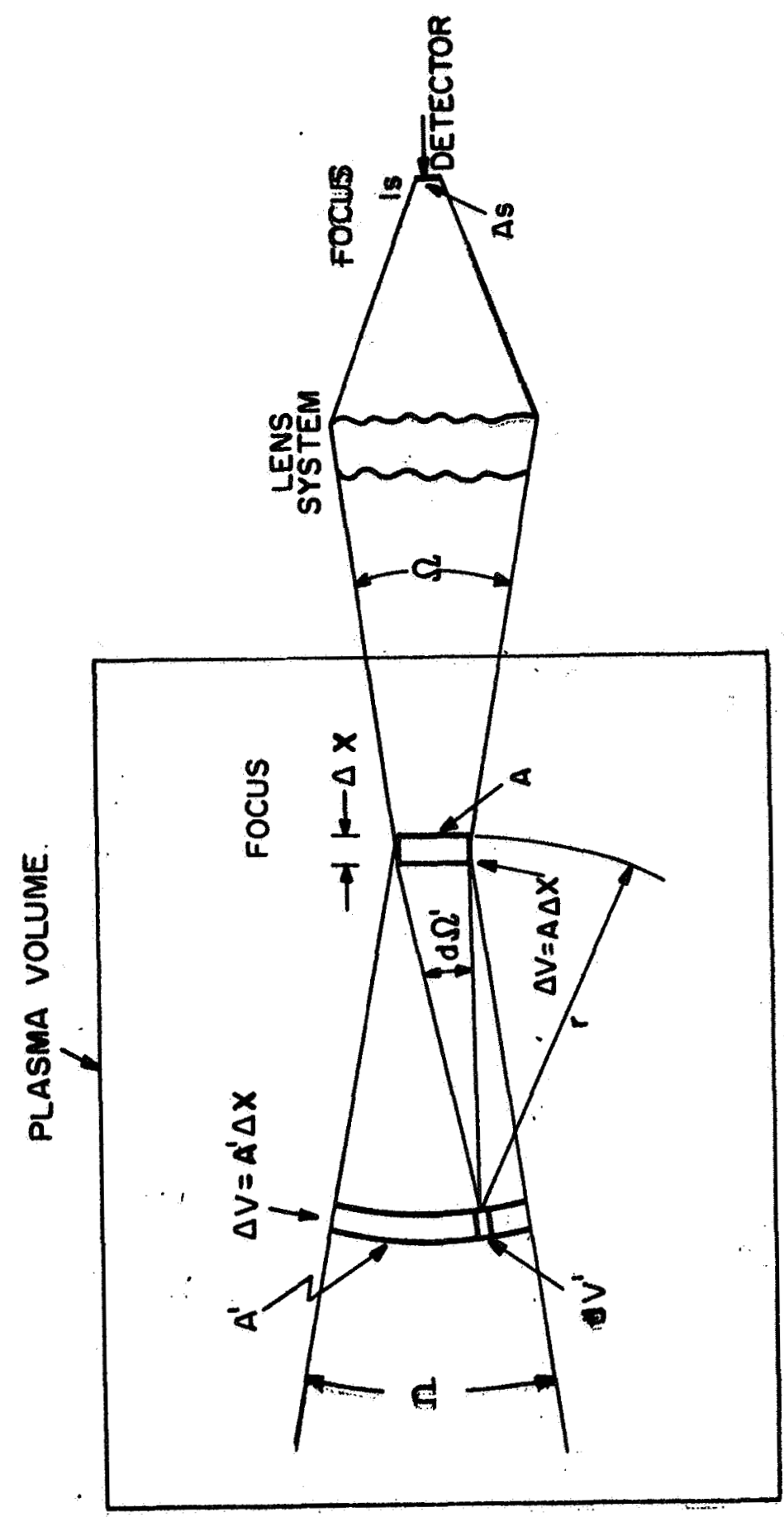

Fig. A-1 Schematic for Calculation of Radiation From a Blasma, Drawn Out of Scale, But A and As are Assimed Macroscop1c. 
plasma, and that $I_{S}$ was at a focal point. Neither restriction is necessary, so $I_{S}=A l$ is quite generally true, regardless of the placement of the detector or whether the focus lies within the plasma. 


\section{APPENDIX B}

The optical depth, D, for line radiation in a plasma as given by Griem ${ }^{16}$ is

$$
D=\frac{\left(\frac{2 \varepsilon l n_{2}}{M_{c}^{2}}\right)^{I / 2}}{2 \pi r_{o} N_{a} e^{-E / \varepsilon \lambda}} \mathrm{cm}
$$

where $\varepsilon=$ temperature in ev

$$
\begin{aligned}
\mathrm{Mc}^{2}= & \text { Rest energy of the atom in ev } \\
r_{0}= & 2.82 \times 10^{-13} \mathrm{~cm}=\text { classical radius of the electron } \\
\mathrm{f}= & \text { oscillator strength for the particular line in } \\
& \text { question } \\
\mathrm{N}_{\mathrm{a}}= & \text { total number of atoms } / \mathrm{cm}^{3} \\
\mathrm{E}= & \text { energy of the excited state in question; measured } \\
& \text { from the ground state in ev } \\
\lambda= & \text { wavelength of absorbed photon in } \mathrm{cm} .
\end{aligned}
$$

For the purpose of this calculation all parameters were chosen so that the depth would be as short as possible, i.e., the worst case. The above formula is for Doppler broadening only, whereas in general other broadening mechanisms are present causing the depth to be larger. The quantities, $f, N_{a}$, and $\lambda$ were all chosen large for quiescent conditions ahead of the shock, 1.e., $\mathrm{f}=1, \mathrm{~N}_{\mathrm{a}} \cong 10^{16}$ part/cc, $\lambda \approx 7900 \mathrm{Ang}$, and $\varepsilon$ was chosen to be $1.7\left(20,000^{\circ} \mathrm{K}\right)$. The calculated value of $D$ for these conditions is $20 \mathrm{~cm}$. 


\section{APPENDIX C}

In reference to the material of Chapter 6.3 it is desired to determine $\gamma(T)$ for an ideal gas allowing ionization to occur. The energy, $u$, per unit volume of such gas is

$$
u=3 / 2 N k T+N_{e} E
$$

where $N=N_{a}+N_{e}+N_{i}=2 N_{e}+N_{a}$

and it is assumed that the atoms have no excited states [this assumption is actually a good one, because nearly all the atoms are in the ground state through the first ionization, which is the range of interest here], $\mathrm{E}$ is the ionization energy, and the other symbols were previously defined. Using the thermodynamic definition of $\gamma$

$$
\gamma=\frac{C_{P}}{C_{V}}=\frac{d u+d(N k T)}{d u}
$$

and the fact that $d N=d_{e}$ this expression may be put in the form:

$$
\gamma=1+\frac{1}{3 / 2+\frac{E / \varepsilon}{1+\frac{1+f}{\varepsilon f^{\prime}}}}
$$

where $\varepsilon=$ temperature in ev

$$
\begin{aligned}
f & =\text { fractional ionization }=\frac{N_{e}}{N_{O}} \\
N_{0} & =N_{a}+N_{e}=\text { total initial number of atoms } / \mathrm{cm}^{3} \\
f^{\prime} & =d f / d \varepsilon \\
E & =\text { ionization energy in ev. }
\end{aligned}
$$

Using the above equation and the material in Section 3.3 the value of $\gamma(T)$ was determined as shown in Fig. 6.8 . 
REFERENCES

1. Conrad Longmire, Elementary Plasma Physics, (Interscience Publishers, 1963).

2. A. R. Kantrowitz, H. E. Petschek, MHD Characteristics and Shock Waves, (Avco Everett Research Laboratory, Report No. 185, July 1964).

3. R. Taussig, Normal Ionizing Shock Waves, (Cölumbia University Plasma Laboratory, Report No. 12, July 1964).

4. R. Taussig, Normal Ionizing Shock Waves - The Effects of Equilibirum, Dissociation and Ionization, (Columbia University Plasma Laboratory, Report No. 14, August 1964).

5. E. N. Parker, A Quasi-Linear Model of Plasma Shock Structure in a Longitudinal Magnetic Field (Enrico Fermi Institute for Nuclear Studies, 59-56).

6. M. Y. Jaffrin and R. F. Probstein, Phys. Fluids 7, 1658 (1964)

7. J. D. Jukes, J. Fluid Mech. 3, 275 (1957).

8. V. D. Shafranov, Soviet Phys. - JETP 5, 1183 (1957).

9. M. Y. Jaffrin, MIT Fluid Mechs. Lab. Publ. No. 64-9, (October 1964).

10. F. I. Boley, P. R. Forman, Physical Reviews Letters, Vol. 12, 385 (April 1964).

11. W. H. Heiser, Phys. Fluids 7, 143 (Jan 1964).

12. G. C. Vlases, Physical Review Letters, Vol. 12, 43 (Jan 1964).

13. J. M. Wilcox et al., Phys. Fluids 4, 1506 (1961).

14. M. H. Brennan et al., J. Nuclear Energy, Part C, Plasma Phys 5 , $229(1963)$.

15. E. A. McLean et al., Phys. Fluids 3, 843 (1960).

16. H. R. Griem, Plasma Spectroscopy (Mc Graw-Hill, N. Y. 1964).

17. R. C. Elton, Stark Profile Measurement of the Lyman $-\alpha$ and Lyman $-\beta$ Lines of Hydrogen (U.S. Navel Research Laboratory No. 5967, July 17, 1963). 
18. Euler, I. Annalen Der Physik 6 , 203 (1953).

19. M. R. Null, W. W. Lozier, J. Opt. Soc. Am. 52, 1156 (1962).

20. W. S. Cooper, An Experimental Investigation of the State of a Highly Ionized Hydrogen Plasma, (Ph.D. Thesis, University of Califormia UCRI-10849).

21. Lyman Spitzer, Physics of Fully Ionized Gases, (Interscience Publishers 1962). 